\title{
Structures and Properties of Known and Postulated Interstellar Cations
}

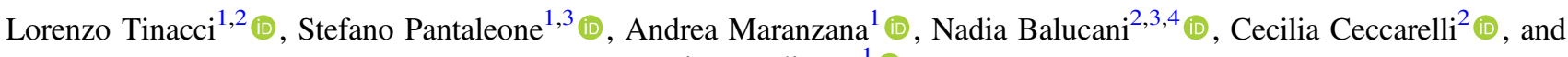 \\ Piero Ugliengo ${ }^{1}$ (i) \\ ${ }^{1}$ Dipartimento di Chimica and Nanostructured Interfaces and Surfaces (NIS) Centre, Universitá degli Studi di Torino, via P. Giuria 7, I-10125 Torino, Italy \\ Lorenzo.Tinacci@univ-grenoble-alpes.fr \\ ${ }^{2}$ Université Grenoble Alpes, CNRS, IPAG, F-38000 Grenoble, France \\ ${ }^{3}$ Dipartimento di Chimica, Biologia e Biotecnologie, Universitá di Perugia, I-06123 Perugia, Italy \\ ${ }^{4}$ Osservatorio Astrofisico di Arcetri, Largo E. Fermi 5, I-50125 Firenze, Italy \\ Received 2021 May 12; revised 2021 July 14; accepted 2021 July 18; published 2021 September 28
}

\begin{abstract}
Positive ions play a fundamental role in interstellar chemistry, especially in cold environments where chemistry is believed to be mainly ion driven. However, in contrast with neutral species, most of the cations present in the astrochemical reaction networks are not fully characterized in the astrochemical literature. To fill this gap, we have carried out new accurate quantum chemical calculations to identify the structures and energies of 262 cations with up to 14 atoms that are postulated to have a role in interstellar chemistry. Optimized structures and rotational constants were obtained at the M06-2X/cc-pVTZ level, while electric dipoles and total electronic energies were computed with CCSD(T)/aug-cc-pVTZ//M06-2X/cc-pVTZ single-point energy calculations. The present work complements the study by Woon \& Herbst, who characterized the structure and energies of 200 neutral species also involved in interstellar chemistry. Taken together, the two data sets can be used to estimate whether a reaction, postulated in present astrochemical reaction networks, is feasible from a thermochemistry point of view and, consequently, to improve the reliability of the present networks used to simulate the interstellar chemistry. We provide an actual example of the potential use of the cations plus neutral data sets. It shows that two reactions, involving Si-bearing ions and present in the widely used reaction networks KIDA and UMIST, cannot occur in the cold interstellar medium because they are endothermic.
\end{abstract}

Unified Astronomy Thesaurus concepts: Astrochemistry (75); Interstellar molecules (849)

Supporting material: data behind figure, machine-readable table

\section{Introduction}

Soon after the first detection in the late 1960s of polyatomic molecules in interstellar cold (10-20 K) molecular clouds (Cheung et al. 1968, 1969; Snyder et al. 1969), the dominant role of cations in the chemistry leading to them became clear (Watson 1973; Herbst \& Klemperer 1973). The reason is relatively simple: molecular clouds are too cold for reactions that present activation barriers to take place, and the vast majority of neutral-neutral reactions possess activation barriers (insurmountable at $10 \mathrm{~K}$ ). Therefore, in cold molecular clouds, chemistry is believed to be mainly driven by cations, whose root is the ionization of hydrogen (both in the atomic and molecular forms) by the cosmic rays that permeate the Milky Way.

The first confirmation of this theoretical prediction came with the detection of $\mathrm{HCO}^{+}$by Snyder et al. (1976). ${ }^{5}$ To date, out of slightly more than 200 interstellar detected species, about 30 are cations, ${ }^{6}$ the last ones discovered being $\mathrm{HC}_{3} \mathrm{~S}^{+}$ and $\mathrm{CH}_{3} \mathrm{CO}^{+}$(Cernicharo et al. 2021a, 2021b). Interestingly, all the 13 detected cations with more than three atoms are, so far, protonated forms of stable and abundant molecules. It is important to emphasize that the relatively low number of detected cations is not due to their real paucity, at least based on the astrochemical theoretical predictions, but on their low abundance and the difficulty of deriving their spectroscopic properties (e.g., McGuire et al. 2020).

\footnotetext{
5 Actually, the theory followed the suggestion by Klemperer (1970) that an unidentified line observed in a few sources was to be attributed to $\mathrm{HCO}^{+}$.

6 https://cdms.astro.uni-koeln.de/classic/molecules
}

As a matter of fact, of the about 500 species involved in the present astrochemical gas-phase reaction networks (e.g., $\mathrm{KIDA}^{7}$ and UMIST), more than half are cations. In the same vein, of the 8000 or so reactions in the same reaction networks, the majority, about 5500, involve cations. Yet, despite the obvious importance of cations in the modeling of interstellar chemistry, no systematic study exists in the literature on the structure and energy of the cations involved in these networks. Indeed, it is worth noting that the abovementioned reaction networks list cations whose structure has seldom been characterized and often appear as chemical formulae guessed on the basis of the reactions that involve them. In contrast, a systematic theoretical study of many neutral species present in the astrochemical reaction networks was carried out more than a decade ago by Woon \& Herbst (2009).

The goal of the present work is to provide accurate physicochemical data for cation species, comparable in terms of methodology with those available for neutral species, to ultimately improve the accuracy of the astrochemical models. An obvious example of the impact of having reliable data of all species present in the astrochemical networks is that this will allow for quick verification of the exo-/endothermicity of the reaction, and, if relevant, excluding it from the network without the need for the very time-consuming characterization of the transition states of the reaction.

To reach the goal of providing reliable data on the cations, accurate estimates of the electronic spin multiplicity,

\footnotetext{
7http://kida.astrophy.u-bordeaux.fr/

8 http://udfa.ajmarkwick.net/
} 
geometrical structure, and absolute electronic energy of each cation are needed (Herzberg 1966; Lattelais et al. 2009, 2010; Chabot et al. 2013). Here we present new computations of the physicochemical properties of the 262 cations present in the KIDA astrochemical gas-phase reaction network (Wakelam et al. 2012).

The article is organized as follows. In Section 2, we provide details on the adopted computational methodology. In Section 3, we report the results of the new computations. In Section 4, we provide two examples of the possible application of the two data sets (the neutral one from Woon \& Herbst (2009) and a cationic one from the present work) to identify and consequently exclude endothermic reactions present in the KIDA and UMIST reaction networks. Section 5 concludes the article and includes the hyperlink to the online database from this work, which is publicly available.

\section{Methodology}

\subsection{Initial Guessed Geometrical Structures}

In the astrochemical reaction networks, cations fall into two general classes of whether or not they are produced by ionization of a mother neutral species. Therefore, we adopted two different approaches to recover the initial guessed structures of the 262 cations.

For the 128 cations belonging to the first class (i.e., from ionization of a mother species), we started from the structure of the neutral species calculated by Woon \& Herbst (2009), removing one electron and then optimizing the structure after assigning the proper charge and spin multiplicity. The reason behind this choice is that although cations are not produced by electron abstraction processes in most cases, astrochemical networks usually postulate that cation structures have the same connectivity of their neutral counterparts.

For about two-thirds of the remaining 134 cations, we retrieved the starting structures from the KIDA database ${ }^{7}$ and the NIST Computational Chemistry Comparison and Benchmark DataBase (CCCBD). ${ }^{9}$ Finally, when only the brute formulas were available (about 50 cations), we guessed the starting structure case by case, looking at the products of the reactions forming and destroying the cations. To automatize the initial geometric guess for the unknown chemical structure, we developed a graph-theory-based software tool coupled with the Universal Force Field (Rappé et al. 1992) implemented in RdKit (Landrum 2016). The script can be found in the online-only material and at the Astro-Chemistry Origin (ACO) Cations Scripts website. ${ }^{10}$

We emphasize that the procedures and choices described above stem from the fact that, very often, only simple connectivity or a cation name are available in the reaction networks and not the structure itself. In the few uncertain cases where cis-trans isomers are possible and no further information is available in the network to differentiate them, we assumed the most stable one (in the specific case, the trans isomer, based on the general rules of organic chemistry).

\footnotetext{
9 http://cccbdb.nist.gov/

10 aco-itn.oapd.inaf.it/aco-public-datasets/theoretical-chemistry-calculations / software-packages/cations-structures-scripts
}

\subsection{Computational Details}

Once the 262 guessed geometrical structures were obtained, a sequence of geometric optimizations at the density functional theory (DFT) level were made, considering both electronic spin multiplicities in the ground and first excited states.

All calculations were carried out with the Gaussian16 program (Frisch et al. 2016). For the DFT calculations, we adopted the Minnesota method M06-2X (Zhao \& Truhlar 2008) coupled with the triple- $\zeta$ Dunning's correlation consistent basis set (cc-pVTZ) (Kendall 1992; Woon \& Dunning 1993) for geometry optimization. We kept the default values set up in Gaussian16 for: the DFT integration grid (i.e., 99,590 grid points), self-consistent field convergence (i.e., $\Delta E=10^{-8} \mathrm{Ha}$ on the rms density matrix and $\Delta E=10^{-6} \mathrm{Ha}$ on the maximum density matrix value and total energy), and geometry optimization tolerances (i.e., $3 \times 10^{-4} \mathrm{Ha}$ and $1.2 \times 10^{-3} \mathrm{a}_{0}$, on rms gradients and displacements, respectively). We carefully explored symmetry constraints to maximize the number of symmetry elements compatible with the most stable structure. Harmonic frequency calculations were performed for all considered cases to ensure that each structure was a minimum of the potential energy surface (PES). Dipole moments and absolute electronic energies were refined at the coupled-cluster level with full single and double excitations and a perturbative treatment of triple excitations $(\mathrm{CCSD}(\mathrm{T})$ and ROCCSD(T); Knowles et al. 1993; Watts et al. 1993) in conjunction with an augmented triple- $\zeta$ correlation consistent basis set (aug-cc-pVTZ; Kendall 1992).

Since unrestricted electronic solutions are affected by spin contamination (i.e., the artificial mixing of different spin states) and this contribution is not negligible nor automatically corrected in Gaussian16 via the spin annihilation procedure, we adopted the Restricted Open (RO) formalism (except for singlet open, since the RO formalism is not applicable), whose wave function is the eigenfunction of the $\hat{S}^{2}$ operator, for all open shell configurations. Spin contamination causes problems in recovering dynamic correlation mainly in post-HF methods that are based on many-body perturbation theory (MP2, CCSD), because the perturbation through high-spin states is too large to be correctly accounted for by these methods (Watts et al. 1993).

Moreover, a stability analysis on the converged wave function was applied to the singlet states computed via the M06-2X/cc-pVTZ level (Bauernschmitt \& Ahlrichs 1996) using the specific keywords $($ opt $=$ stable $)$ provided in Gaussian16 (see Figure 1; $\Psi_{1 c}$ stability block).

The scheme summarizing the adopted strategy is shown in Figure 1, where the various steps needed to reach the final minimum structure are shown.

Rendering of molecule images have been obtained via the VMD software (Humphrey et al. 1996), and the graphics elaboration and plots via the TikZ and PGFPlots LATEX packages.

\subsection{Benchmark Method}

In order to test the accuracy of the above-described methodology, a benchmark on both structure and wave function optimizations using different methods was carried out.

Geometry optimization-We checked the M06-2X/cc-pVTZ level of theory by comparing our results obtained for a subset of molecules with those computed at the CCSD/aug-cc-pVTZ level, as shown in Table 1 . Both the geometry rms deviation (RMSD) and the energy difference are small enough (less than $\sim 0.045 \AA$ 


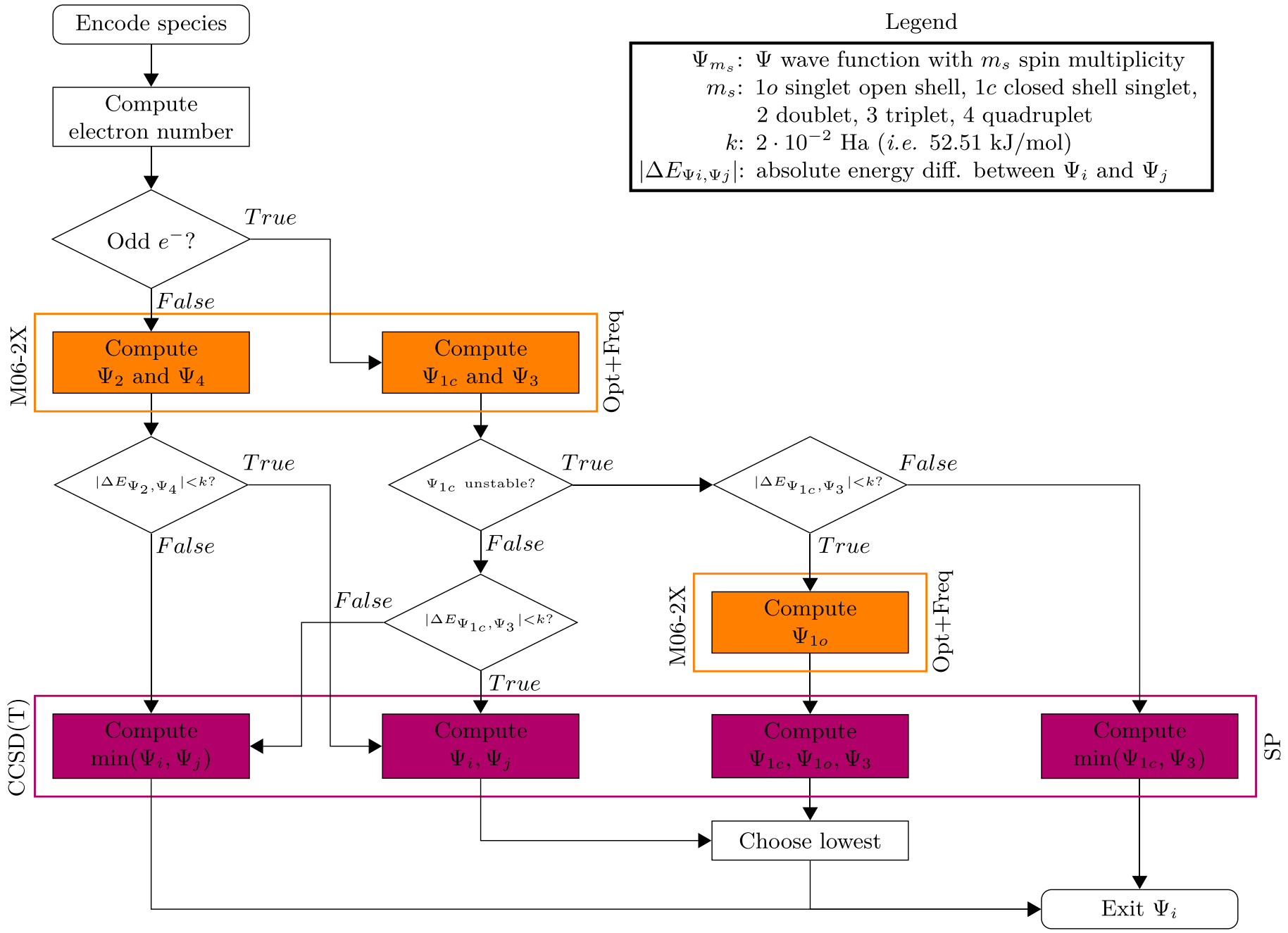

Figure 1. Adopted procedure to define the ground electronic state for a species and to achieve the related optimized geometry and cation properties. To this end, we used two levels of theory: M06-2X/cc-pVTZ and CCSD(T)/aug-cc-pVTZ. In the M06-2X block, a geometric optimization (Opt) and vibrational frequencies calculation (Freq) are performed to ensure that a minimum PES is achieved. In the CCSD(T) block, the final energy is then refined as a single-point evaluation at the $\operatorname{CCSD}(\mathrm{T})$ level together with the corresponding properties. The $\Psi_{1 c}$ stability is tested with the Gaussian 16 wave function stability tool to find restricted $\rightarrow$ unrestricted wave function instability.

and $\sim 1.7 \mathrm{~kJ} \mathrm{~mol}^{-1}$, respectively) to validate the present methodology. Moreover, the average $\Delta E$ is $0.522 \mathrm{~kJ} \mathrm{~mol}^{-1}$, which is lower than the commonly accepted quantum mechanics calculation accuracy of $\sim 4 \mathrm{~kJ} \mathrm{~mol}^{-1}$ (i.e., $1 \mathrm{kcal} \mathrm{mol}^{-1}$ ). The extended internal coordinates geometry optimization comparison is available in the Appendix.

Wave function optimization-The procedure shown in Figure 1 was first tested on two very common molecules: ethylene $\left(\mathrm{C}_{2} \mathrm{H}_{6}\right)$ and methylene $\left(\mathrm{CH}_{2}\right)$. These molecules present different ground states, i.e., singlet closed shell $\left({ }^{1} \mathrm{~A}_{g}\right)$ and triplet $\left({ }^{3} \mathrm{~B}_{1}\right)$ for ethylene and methylene, respectively. Their corresponding excited states are triplet $\left({ }^{3} \mathrm{~A}_{1}\right)$ for ethylene and singlet closed shell $\left({ }^{1} \mathrm{~A}_{1}\right)$ for methylene. Our calculated transition energies are in good agreement with the experimental data: 282 versus $272 \mathrm{~kJ} \mathrm{~mol}^{-1}$ (Douglas et al. 1955) and 40 versus $38 \mathrm{~kJ}$ $\mathrm{mol}^{-1}$ (Shavitt 1985) for ethylene and methylene, respectively. For the singlet closed shell methylene $\left({ }^{1} \mathrm{~A}_{1}\right)$ the stability analysis was also performed revealing a preference for the unrestricted solution with respect to the restricted one, as expected.

Dipole moment evaluation-Woon \& Herbst (2009) showed that the $\operatorname{CCSD(T)/aug-cc-pVTZ~electronic~dipole~for~neutral~}$ species is in good agreement with the experimental data. We
Table 1

Rms Displacement (RMSD) of Atomic Positions and the Absolute Energy Difference

\begin{tabular}{lccc}
\hline \hline Species & State & $\begin{array}{c}\Delta E \\
{\left[\mathrm{~kJ} \mathrm{~mol}^{-1}\right]}\end{array}$ & $\begin{array}{c}\text { RMSD } \\
{[\AA]}\end{array}$ \\
\hline $\mathrm{C}_{2}^{+}$ & ${ }^{4} \Sigma_{g}$ & 0.247 & 0.003 \\
$\mathrm{NH}_{4}^{+}$ & ${ }^{1} \mathrm{~A}_{1}$ & -0.022 & 0.000 \\
$\mathrm{H}_{2} \mathrm{CO}^{+}$ & ${ }^{2} \mathrm{~B}_{2}$ & 0.247 & 0.007 \\
$\mathrm{PNH}_{2}^{+}$ & ${ }^{2} \mathrm{~B}_{2}$ & 0.512 & 0.005 \\
$1-\mathrm{C}_{3} \mathrm{H}_{2}^{+}$ & ${ }^{2} \mathrm{~A}^{\prime}$ & 0.055 & 0.045 \\
$\mathrm{H}_{3} \mathrm{CS}^{+}$ & ${ }^{3} \mathrm{~A}_{1}$ & 0.509 & 0.012 \\
$\mathrm{c}^{-} \mathrm{C}_{3} \mathrm{H}_{3}^{+}$ & ${ }^{1} \mathrm{~A}_{1}{ }^{+}$ & 0.714 & 0.004 \\
$\mathrm{C}_{4} \mathrm{H}_{3}^{+}$ & ${ }^{1} \mathrm{~A}_{1}$ & 1.681 & 0.008 \\
$\mathrm{CH}_{3} \mathrm{CHOH}^{+}$ & ${ }^{1} \mathrm{~A}^{\prime}$ & 0.432 & 0.006 \\
$\mathrm{H}_{2} \mathrm{C}_{3} \mathrm{O}^{+}$ & ${ }^{2} \mathrm{~B}_{2}$ & 0.845 & 0.005 \\
\hline
\end{tabular}

Note. Calculated at $\operatorname{CCSD}(\mathrm{T}) /$ aug-cc-pVTZ//M06-2X/cc-pVTZ compared with calculations at $\operatorname{CCSD}(\mathrm{T}) /$ aug-cc-pVTZ//CCSD/aug-cc-pVTZ.

expect a similar or better agreement for cations, in virtue of the more contracted nature of the electron density compared to the more diffuse one in neutral species. 

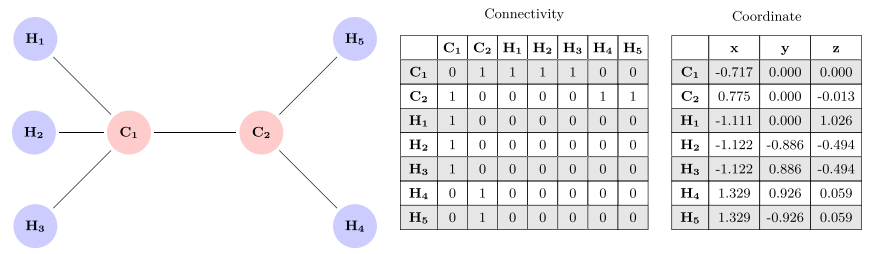

(a) $\mathrm{C}_{2} \mathrm{H}_{5}$
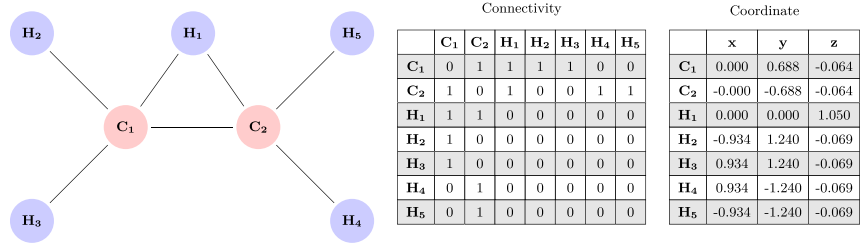

(b) $\mathrm{C}_{2} \mathrm{H}_{5}^{+}$

Figure 2. Graphs, connectivity matrices, and coordinates (in $\AA$ ) of the neutral ethyl radical $\mathrm{C}_{2} \mathrm{H}_{5}$ and its cation $\mathrm{C}_{2} \mathrm{H}_{5}^{+}$.

\subsection{Neutral versus Cation Structure Connectivity}

Since many cation structures are derived from the neutral counterpart, we have calculated the ionization energy and followed the connectivity change (if any) after the geometry relaxation on the proper PES. In order to check if a cation retains the same connectivity of the neutral counterpart after geometric relaxation, the graph theory approach was used. Finally, the adiabatic ionization energy for the species that have a neutral counterpart will be presented in the dedicated subsection 3.2 with a comparison with available experimental data.

First, all the coordinate files are converted into chemical graphs (Trinajstic 2018) using the covalent radii and distance functions implemented in the Atomic Simulation Environment (ASE) python package (Larsen et al. 2017). A chemical graph is a nondirected graph where atoms and bonds in molecules correspond to nodes and edges, respectively. The different chemical elements present in the periodic table are represented in graphs as colors assigned to the vertices: the graph is, therefore, defined as a multicolored graph. Second, the graphs related to the neutral and ionized molecules are tested for isomorphism. Two graphs which contain the same number of colored vertices connected in the same way are considered isomorphic. The NetworkX python package (Hagberg et al. 2008) was used in order to deal with graph objects. Examples of chemical graphs are shown in Figure 2.

\section{Results}

The structures of the cations computed following the procedures and methodology described in the previous section are minima of PES when starting from the guessed ones, i.e., we did not explore the full PES in search of the global minimum. Indeed, our goal is to consider cations whose connectivity derives from the astrochemical reaction networks with structures based on the reactions giving rise to their formation. When more isomeric forms exist, we adopted the most stable one when specific information in the reaction network was missing. Finally, we cross-checked our computed cation structures with the literature ones, in the relatively few cases where they are available, and generally found a very good agreement.
Table 2

Predicted Equilibrium Structures and Properties of Diatomic Species

\begin{tabular}{|c|c|c|c|c|c|c|}
\hline Species & State & Symmetry & $\mu$ & $B_{e}$ & $r_{e}$ & $r_{e}(\exp )$ \\
\hline$\overline{\mathrm{H}_{2}^{+}}$ & ${ }^{2} \Sigma_{g}$ & $\mathrm{D}_{\infty h}$ & 0.000 & 827.202 & 1.101 & $1.057^{\mathrm{a}}$ \\
\hline $\mathrm{HeH}^{+}$ & ${ }^{1} \Sigma$ & $\mathrm{C}_{\infty v}$ & 1.343 & 1010.927 & 0.788 & \\
\hline $\mathrm{CH}^{+}$ & ${ }^{1} \Sigma$ & $\mathrm{C}_{\infty v}$ & 1.565 & 428.701 & 1.126 & $1.131^{\mathrm{b}}$ \\
\hline $\mathrm{NH}^{+}$ & ${ }^{2} \Pi$ & $\mathrm{C}_{\infty v}$ & 1.834 & 466.167 & 1.074 & \\
\hline $\mathrm{OH}^{+}$ & ${ }^{3} \Sigma$ & $\mathrm{C}_{\infty v}$ & 2.127 & 501.498 & 1.031 & $1.029^{\mathrm{a}}$ \\
\hline $\mathrm{HF}^{+}$ & ${ }^{2} \Pi$ & $\mathrm{C}_{\infty v}$ & 2.478 & 518.136 & 1.010 & \\
\hline $\mathrm{C}_{2}^{+}$ & ${ }^{4} \Sigma_{g}$ & $\mathrm{D}_{\infty h}$ & 0.000 & 43.077 & 1.398 & \\
\hline $\mathrm{CN}^{+}$ & ${ }^{1} \Sigma$ & $\mathrm{C}_{\infty v}$ & 2.846 & 57.192 & 1.169 & $1.290^{\mathrm{a}}$ \\
\hline $\mathrm{CO}^{+}$ & ${ }^{2} \Sigma$ & $\mathrm{C}_{\infty v}$ & 3.286 & 60.629 & 1.103 & \\
\hline $\mathrm{N}_{2}^{+}$ & ${ }^{2} \Sigma_{g}$ & $\mathrm{D}_{\infty h}$ & 0.000 & 59.719 & 1.099 & $1.113^{\mathrm{a}}$ \\
\hline $\mathrm{SiH}^{+}$ & ${ }^{1} \Sigma$ & $\mathrm{C}_{\infty v}$ & 0.330 & 228.988 & 1.506 & $1.499^{\mathrm{a}}$ \\
\hline $\mathrm{NO}^{+}$ & ${ }^{1} \Sigma$ & $\mathrm{C}_{\infty v}$ & 0.603 & 61.361 & 1.050 & $1.062^{\mathrm{a}}$ \\
\hline $\mathrm{CF}^{+}$ & ${ }^{1} \Sigma$ & $\mathrm{C}_{\infty v}$ & 1.305 & 51.829 & 1.151 & $1.263^{\mathrm{a}}$ \\
\hline $\mathrm{PH}^{+}$ & ${ }^{2} \Pi$ & $\mathrm{C}_{\infty v}$ & 0.741 & 255.260 & 1.424 & \\
\hline $\mathrm{O}_{2}^{+}$ & ${ }^{2} \Pi_{g}$ & $\mathrm{D}_{\infty h}$ & 0.000 & 52.927 & 1.093 & $1.116^{\mathrm{a}}$ \\
\hline $\mathrm{HS}^{+}$ & ${ }^{3} \Sigma$ & $\mathrm{C}_{\infty v}$ & 1.170 & 278.193 & 1.364 & \\
\hline $\mathrm{HCl}^{+}$ & ${ }^{2} \Pi$ & $\mathrm{C}_{\infty v}$ & 1.634 & 295.614 & 1.321 & \\
\hline $\mathrm{SiC}^{+}$ & ${ }^{4} \Sigma$ & $\mathrm{C}_{\infty v}$ & 1.026 & 18.276 & 1.815 & \\
\hline $\mathrm{SiN}^{+}$ & ${ }^{3} \Pi$ & $\mathrm{C}_{\infty v}$ & 2.860 & 17.154 & 1.777 & \\
\hline $\mathrm{CP}^{+}$ & ${ }^{3} \Sigma$ & $\mathrm{C}_{\infty v}$ & 0.120 & 22.542 & 1.610 & \\
\hline $\mathrm{CS}^{+}$ & ${ }^{2} \Sigma$ & $\mathrm{C}_{\infty v}$ & 0.945 & 26.449 & 1.480 & \\
\hline $\mathrm{PN}^{+}$ & ${ }^{2} \Sigma$ & $\mathrm{C}_{\infty v}$ & 2.040 & 24.210 & 1.471 & \\
\hline $\mathrm{NS}^{+}$ & ${ }^{1} \Sigma$ & $\mathrm{C}_{\infty v}$ & 2.209 & 25.735 & 1.420 & \\
\hline $\mathrm{PO}^{+}$ & ${ }^{1} \Sigma$ & $\mathrm{C}_{\infty v}$ & 4.030 & 23.802 & 1.419 & \\
\hline $\mathrm{SiF}^{+}$ & ${ }^{1} \Sigma$ & $\mathrm{C}_{\infty v}$ & 3.599 & 18.802 & 1.541 & \\
\hline $\mathrm{CCl}^{+}$ & ${ }^{1} \Sigma$ & $\mathrm{C}_{\infty v}$ & 0.165 & 24.050 & 1.534 & \\
\hline $\mathrm{SiO}^{+}$ & ${ }^{2} \Sigma$ & $\mathrm{C}_{\infty v}$ & 3.253 & 21.372 & 1.524 & \\
\hline $\mathrm{SO}^{+}$ & ${ }^{2} \Pi$ & $\mathrm{C}_{\infty v}$ & 2.785 & 23.644 & 1.416 & \\
\hline $\mathrm{ClO}^{+}$ & ${ }^{3} \Sigma$ & $\mathrm{C}_{\infty v}$ & 0.569 & 21.249 & 1.472 & \\
\hline $\mathrm{SiS}^{+}$ & ${ }^{2} \Sigma$ & $\mathrm{C}_{\infty v}$ & 4.576 & 9.240 & 1.915 & \\
\hline $\mathrm{S}_{2}^{+}$ & ${ }^{2} \Pi_{g}$ & $\mathrm{D}_{\infty h}$ & 0.000 & 9.641 & 1.811 & \\
\hline
\end{tabular}

Notes. $\mu$ is the electric dipole moment in Debye units. $B_{e}$ is the rotational constant expressed in gigahertz referring to the equilibrium structure. $r_{e}$ is the calculated internuclear equilibrium distance expressed in angstroms, meanwhile $r_{e}(\exp )$ are the available experimental data.

a From Chase (1996).

${ }^{\mathrm{b}}$ From Huber (2013).

\subsection{Cations Properties and Geometries}

Tables 2-6 list the calculated properties of the 262 cations studied in this work. In the tables, we grouped the cations into five categories: (1) diatomic species (Table 2); (2) linear species with 3-12 atoms (Table 3); (3) $\mathrm{C}_{2 v}$ symmetry species (Table 4); (4) planar species (excluding those belonging to point 3) (Table 5); (5) nonplanar species (Table 6). Within each table, cations are ordered by increasing number of atoms and, within each subset, by increasing molecular mass. A sample of the derived structures is shown in Figure 3.

Online-only material with extended information on all 262 cations is provided as data behind Figure 3. The center of the coordinate frame with respect to the dipole moment components is referred to is the center of nuclear charge. We also make the data publicly available on the website of the ACO project site. ${ }^{11}$ The web-based ACO cation structure database ${ }^{12}$ is based on the molecule hyperactive $\mathrm{JSmol}^{13}$ plugin. The ACO

\footnotetext{
11 https://aco-itn.oapd.inaf.it/home

12 aco-itn.oapd.inaf.it/aco-public-datasets/theoretical-chemistry-calculations / cations-database

${ }^{13} \mathrm{JSmol}$ is an open-source Java viewer for chemical structures in 3D (http:// www.jmol.org/).
} 
Table 3

Predicted Equilibrium Properties of Linear Polyatomic Species

\begin{tabular}{|c|c|c|c|c|c|c|c|c|c|}
\hline Species & State & Symmetry & $\mu$ & $B_{e}$ & Species & State & Symmetry & $\mu$ & $\overline{B_{e}}$ \\
\hline$\overline{\mathrm{C}_{2} \mathrm{H}^{+}}$ & ${ }^{3} \Pi$ & $\mathrm{C}_{\infty v}$ & 0.755 & 41.665 & $\mathrm{SiC}_{4}^{+}$ & ${ }^{2} \Sigma$ & $\mathrm{C}_{\infty v}$ & 7.013 & $\overline{1.516}$ \\
\hline $\mathrm{HCN}^{+}$ & ${ }^{2} \Pi$ & $\mathrm{C}_{\infty v}$ & 3.586 & 41.202 & $\mathrm{C}_{4} \mathrm{P}^{+}$ & ${ }^{1} \Sigma$ & $\mathrm{C}_{\infty v}$ & 2.101 & 1.530 \\
\hline $\mathrm{HNC}^{+}$ & ${ }^{2} \Sigma$ & $\mathrm{C}_{\infty v}$ & 0.148 & 47.950 & $\mathrm{C}_{4} \mathrm{~S}^{+}$ & ${ }^{2} \Pi$ & $\mathrm{C}_{\infty v}$ & 2.294 & 1.531 \\
\hline $\mathrm{HCO}^{+}$ & ${ }^{1} \Sigma$ & $\mathrm{C}_{\infty v}$ & 4.172 & 45.359 & $\mathrm{C}_{4} \mathrm{H}_{2}^{+}$ & ${ }^{2} \Pi_{g}$ & $\mathrm{D}_{\infty h}$ & 0.000 & 4.431 \\
\hline $\mathrm{HOC}^{+}$ & ${ }^{1} \Sigma$ & $\mathrm{C}_{\infty v}$ & 2.380 & 44.893 & $\mathrm{HC}_{2} \mathrm{NCH}^{+}$ & ${ }^{1} \Sigma$ & $\mathrm{C}_{\infty v}$ & 3.484 & 4.689 \\
\hline $\mathrm{N}_{2} \mathrm{H}^{+}$ & ${ }^{1} \Sigma$ & $\mathrm{C}_{\infty v}$ & 3.154 & 47.597 & $\mathrm{HC}_{3} \mathrm{NH}^{+}$ & ${ }^{1} \Sigma$ & $\mathrm{C}_{\infty v}$ & 1.246 & 4.350 \\
\hline $\mathrm{CNC}^{+}$ & ${ }^{1} \Sigma_{g}$ & $\mathrm{D}_{\infty h}$ & 0.000 & 13.661 & $\mathrm{C}_{5} \mathrm{H}^{+}$ & ${ }^{1} \Sigma$ & $\mathrm{C}_{\infty v}$ & 2.677 & 2.420 \\
\hline $\mathrm{C}_{2} \mathrm{~N}^{+}$ & ${ }^{1} \Sigma$ & $\mathrm{C}_{\infty v}$ & 2.660 & 12.004 & $\mathrm{HC}_{4} \mathrm{~N}^{+}$ & ${ }^{2} \Pi$ & $\mathrm{C}_{\infty v}$ & 6.436 & 2.327 \\
\hline $\mathrm{CHSi}^{+}$ & ${ }^{3} \Sigma$ & $\mathrm{C}_{\infty v}$ & 0.246 & 15.767 & $\mathrm{HC}_{4} \mathrm{O}^{+}$ & ${ }^{3} \Sigma$ & $\mathrm{C}_{\infty v}$ & 4.504 & 2.246 \\
\hline $\mathrm{NCO}^{+}$ & ${ }^{3} \Sigma$ & $\mathrm{C}_{\infty v}$ & 1.117 & 11.196 & $\mathrm{C}_{6}^{+}$ & ${ }^{2} \Pi_{u}$ & $\mathrm{D}_{\infty h}$ & 0.000 & 1.445 \\
\hline $\mathrm{HNSi}^{+}$ & ${ }^{2} \Sigma$ & $\mathrm{C}_{\infty v}$ & 3.821 & 16.886 & $\mathrm{C}_{5} \mathrm{~N}^{+}$ & ${ }^{3} \Sigma$ & $\mathrm{C}_{\infty v}$ & 3.890 & 1.391 \\
\hline $\mathrm{HCP}^{+}$ & ${ }^{2} \Pi$ & $\mathrm{C}_{\infty v}$ & 0.985 & 18.914 & $\mathrm{SiC}_{4} \mathrm{H}^{+}$ & ${ }^{1} \Sigma$ & $\mathrm{C}_{\infty v}$ & 2.487 & 1.440 \\
\hline $\mathrm{CO}_{2}^{+}$ & ${ }^{2} \Pi_{g}$ & $\mathrm{D}_{\infty h}$ & 0.000 & 11.600 & $\mathrm{PC}_{4} \mathrm{H}^{+}$ & ${ }^{2} \Sigma$ & $\mathrm{C}_{\infty v}$ & 0.274 & 1.444 \\
\hline $\mathrm{HCS}^{+}$ & ${ }^{1} \Sigma$ & $\mathrm{C}_{\infty v}$ & 1.964 & 21.653 & $\mathrm{HC}_{4} \mathrm{~S}^{+}$ & ${ }^{3} \Sigma$ & $\mathrm{C}_{\infty v}$ & 2.736 & 1.463 \\
\hline $\mathrm{HSiO}^{+}$ & ${ }^{1} \Sigma$ & $\mathrm{C}_{\infty v}$ & 6.769 & 19.171 & $\mathrm{C}_{6} \mathrm{H}^{+}$ & ${ }^{3} \Sigma$ & $\mathrm{C}_{\infty v}$ & 3.410 & 1.393 \\
\hline $\mathrm{HPN}^{+}$ & ${ }^{1} \Sigma$ & $\mathrm{C}_{\infty v}$ & 0.267 & 20.749 & $\mathrm{HC}_{5} \mathrm{~N}^{+}$ & ${ }^{2} \Pi$ & $\mathrm{C}_{\infty v}$ & 7.395 & 1.345 \\
\hline $\mathrm{NO}_{2}^{+}$ & ${ }^{1} \Sigma_{g}$ & $\mathrm{D}_{\infty h}$ & 0.000 & 12.903 & $\mathrm{HC}_{5} \mathrm{O}^{+}$ & ${ }^{1} \Sigma$ & $\mathrm{C}_{\infty v}$ & 2.586 & 1.308 \\
\hline $\mathrm{SiC}_{2}^{+}$ & ${ }^{2} \Sigma$ & $\mathrm{C}_{\infty v}$ & 1.472 & 6.125 & $\mathrm{C}_{7}^{+}$ & ${ }^{2} \Sigma_{u}$ & $\mathrm{D}_{\infty h}$ & 0.000 & 0.912 \\
\hline $\mathrm{SiNC}^{+}$ & ${ }^{1} \Sigma$ & $\mathrm{C}_{\infty v}$ & 4.015 & 6.653 & $\mathrm{C}_{6} \mathrm{H}_{2}^{+}$ & ${ }^{2} \Pi_{u}$ & $\mathrm{D}_{\infty h}$ & 0.000 & 1.343 \\
\hline $\mathrm{C}_{2} \mathrm{~S}^{+}$ & ${ }^{2} \Pi$ & $\mathrm{C}_{\infty v}$ & 1.112 & 6.489 & $\mathrm{H}_{2} \mathrm{C}_{5} \mathrm{~N}^{+}$ & ${ }^{1} \Sigma$ & $\mathrm{C}_{\infty v}$ & 3.489 & 1.300 \\
\hline $\mathrm{OCS}^{+}$ & ${ }^{2} \Pi$ & $\mathrm{C}_{\infty v}$ & 1.653 & 5.849 & $\mathrm{C}_{7} \mathrm{H}^{+}$ & ${ }^{1} \Sigma$ & $\mathrm{C}_{\infty v}$ & 2.299 & 0.885 \\
\hline $\mathrm{HSiS}^{+}$ & ${ }^{1} \Sigma$ & $\mathrm{C}_{\infty v}$ & 5.168 & 8.661 & $\mathrm{HC}_{6} \mathrm{~N}^{+}$ & ${ }^{2} \Pi$ & $\mathrm{C}_{\infty v}$ & 7.813 & 0.850 \\
\hline $\mathrm{C}_{2} \mathrm{H}_{2}^{+}$ & ${ }^{2} \Pi_{u}$ & $\mathrm{D}_{\infty h}$ & 0.000 & 33.595 & $\mathrm{C}_{8}^{+}$ & ${ }^{2} \Sigma_{g}$ & $\mathrm{D}_{\infty h}$ & 0.000 & 0.610 \\
\hline $\mathrm{HCNH}^{+}$ & ${ }^{1} \Sigma$ & $\mathrm{C}_{\infty v}$ & 0.566 & 37.543 & $\mathrm{C}_{7} \mathrm{~N}^{+}$ & ${ }^{3} \Sigma$ & $\mathrm{C}_{\infty v}$ & 4.860 & 0.587 \\
\hline $\mathrm{C}_{3} \mathrm{H}^{+}$ & ${ }^{1} \Sigma$ & $\mathrm{C}_{\infty v}$ & 2.552 & 11.262 & $\mathrm{C}_{8} \mathrm{H}^{+}$ & ${ }^{3} \Sigma$ & $\mathrm{C}_{\infty v}$ & 3.461 & 0.592 \\
\hline $\mathrm{HSiNH}^{+}$ & ${ }^{1} \Sigma$ & $\mathrm{C}_{\infty v}$ & 3.460 & 17.494 & $\mathrm{HC}_{7} \mathrm{~N}^{+}$ & ${ }^{2} \Pi$ & $\mathrm{C}_{\infty v}$ & 8.764 & 0.570 \\
\hline $\mathrm{C}_{4}^{+}$ & ${ }^{2} \Pi_{g}$ & $\mathrm{D}_{\infty h}$ & 0.000 & 4.836 & $\mathrm{HC}_{7} \mathrm{O}^{+}$ & ${ }^{1} \Sigma$ & $\mathrm{C}_{\infty v}$ & 1.022 & 0.555 \\
\hline $\mathrm{C}_{3} \mathrm{O}^{+}$ & ${ }^{2} \Sigma$ & $\mathrm{C}_{\infty v}$ & 2.809 & 4.879 & $\mathrm{C}_{9}^{+}$ & ${ }^{2} \Sigma_{u}$ & $\mathrm{C}_{\infty v}$ & 0.011 & 0.429 \\
\hline $\mathrm{C}_{2} \mathrm{~N}_{2}^{+}$ & ${ }^{2} \Pi_{g}$ & $\mathrm{C}_{\infty v}$ & 0.000 & 4.716 & $\mathrm{C}_{8} \mathrm{~N}^{+}$ & ${ }^{1} \Sigma$ & $\mathrm{C}_{\infty v}$ & 7.020 & 0.414 \\
\hline $\mathrm{SiC}_{2} \mathrm{H}^{+}$ & ${ }^{1} \Sigma$ & $\mathrm{C}_{\infty v}$ & 0.623 & 5.582 & $\mathrm{C}_{8} \mathrm{H}_{2}^{+}$ & ${ }^{2} \Pi_{g}$ & $\mathrm{D}_{\infty h}$ & 0.000 & 0.574 \\
\hline $\mathrm{PC}_{2} \mathrm{H}^{+}$ & ${ }^{2} \Pi$ & $\mathrm{C}_{\infty v}$ & 0.219 & 5.803 & $\mathrm{C}_{7} \mathrm{H}_{2} \mathrm{~N}^{+}$ & ${ }^{1}{ }_{\Sigma}^{\circ}$ & $\mathrm{C}_{\infty v}$ & 6.191 & 0.556 \\
\hline $\mathrm{HC}_{2} \mathrm{~S}^{+}$ & ${ }^{3} \Sigma$ & $\mathrm{C}_{\infty v}$ & 2.502 & 6.049 & $\mathrm{C}_{9} \mathrm{H}^{+}$ & ${ }^{1} \Sigma$ & $\mathrm{C}_{\infty v}$ & 1.405 & 0.418 \\
\hline $1-\mathrm{SiC}_{3}^{+}$ & ${ }^{2} \Pi$ & $\mathrm{C}_{\infty v}$ & 1.129 & 2.651 & $\mathrm{HC}_{8} \mathrm{~N}^{+}$ & ${ }^{2} \Pi$ & $\mathrm{C}_{\infty v}$ & 8.870 & 0.403 \\
\hline $\mathrm{C}_{3} \mathrm{~S}^{+}$ & ${ }^{2} \Sigma$ & $\mathrm{C}_{\infty v}$ & 0.962 & 2.858 & $\mathrm{C}_{10}^{+}$ & ${ }^{2} \Pi_{u}$ & $\mathrm{D}_{\infty h}$ & 0.000 & 0.312 \\
\hline $\mathrm{C}_{4} \mathrm{H}^{+}$ & ${ }^{3} \Sigma$ & $\mathrm{C}_{\infty v}$ & 3.140 & 4.640 & $\mathrm{C}_{9} \mathrm{~N}^{+}$ & ${ }^{3} \Sigma$ & $\mathrm{C}_{\infty v}$ & 5.942 & 0.301 \\
\hline $\mathrm{HC}_{3} \mathrm{~N}^{+}$ & ${ }^{2} \Pi$ & $\mathrm{C}_{\infty v}$ & 6.378 & 4.568 & $\mathrm{C}_{10} \mathrm{H}^{+}$ & ${ }^{3} \Sigma$ & $\mathrm{C}_{\infty v}$ & 3.258 & 0.304 \\
\hline $\mathrm{HC}_{3} \mathrm{O}^{+}$ & ${ }^{1} \Sigma$ & $\mathrm{C}_{\infty v}$ & 3.635 & 4.484 & $\mathrm{C}^{\circ} \mathrm{HN}^{+}$ & ${ }^{2} \Pi$ & $\mathrm{C}_{\infty v}$ & 10.189 & 0.294 \\
\hline $\mathrm{C}_{5}^{+}$ & ${ }^{2} \Sigma_{u}$ & $\mathrm{D}_{\infty h}$ & 0.000 & 2.596 & $\mathrm{HC}_{9} \mathrm{O}^{+}$ & ${ }^{1} \Sigma$ & $\mathrm{C}_{\infty v}$ & 1.055 & 0.286 \\
\hline $\mathrm{C}_{4} \mathrm{~N}^{+}$ & ${ }^{1} \Sigma$ & $\mathrm{C}_{\infty v}$ & 3.900 & 2.438 & $\mathrm{C}_{11}^{+}$ & ${ }^{2} \Sigma_{u}$ & $\mathrm{D}_{\infty h}$ & 0.000 & 0.235 \\
\hline $\mathrm{SiC}_{3} \mathrm{H}^{+}$ & ${ }^{3} \Sigma$ & $\mathrm{C}_{\infty v}$ & 0.661 & 2.556 & $\mathrm{C}_{10} \mathrm{~N}^{+}$ & ${ }^{1} \Sigma$ & $\mathrm{C}_{\infty v}$ & 8.995 & 0.227 \\
\hline $\mathrm{PC}_{3} \mathrm{H}^{+}$ & ${ }^{2} \Pi$ & $\mathrm{C}_{\infty v}$ & 1.002 & 2.662 & $\mathrm{C}_{10} \mathrm{H}_{2}^{+}$ & ${ }^{2} \Pi_{u}$ & $\mathrm{D}_{\infty h}$ & 0.000 & 0.296 \\
\hline $\mathrm{HC}_{3} \mathrm{~S}^{+}$ & ${ }^{1} \Sigma$ & $\mathrm{C}_{\infty v}$ & 1.892 & 2.753 & $\mathrm{HC}_{10} \mathrm{~N}^{+}$ & ${ }^{2} \Pi$ & $\mathrm{C}_{\infty v}$ & 9.935 & 0.222 \\
\hline
\end{tabular}

Note. $\mu$ is the electric dipole moment in Debye units. $B_{e}$ is the rotational constant expressed in gigahertz referring to the equilibrium structure.

cation structure website will be periodically updated when new species will be added to the database. On the same site, we also provide the python script tool ${ }^{14}$ to convert molecules into a chemical graph and to exert control if two species share the same connectivity using the isomorphic function. The script can also be found in the online-only material.

Finally, while the rotational constants are reported in Tables 2 and 3 and in the web-based ACO cations database (for all the other nonlinear species), we warn the reader that the level of theory is not sufficiently accurate to allow their use for assigning bands in experimental spectra.

\footnotetext{
14 aco-itn.oapd.inaf.it/aco-public-datasets/theoretical-chemistry-calculations/ software-packages/cations-structures-scripts
}

\subsection{Adiabatic Ionization Energy}

Table 7 shows the adiabatic ionization energies (IE) for neutral species computed at CCSD(T)/aug-cc-pVTZ by Woon \& Herbst (2009) along with those of the cation counterparts. All computed IE are without zero-point energy (ZPE) correction. Note that the cation counterparts are part of the group whose structures are derived from the ionization of neutral species, described in Section 2.1. As some of the data on larger molecules provided by Woon \& Herbst (2009) are computed with a lower-quality basis set, we recomputed the energy for those species with the aug-cc-pVTZ.

The rms error between the computed ionization energies (adiabatic IE) and the experimental available data (vertical IE) 
Table 4

Predicted Equilibrium Properties of Planar Polyatomic Species with $\mathrm{C}_{2 v}$ Symmetry

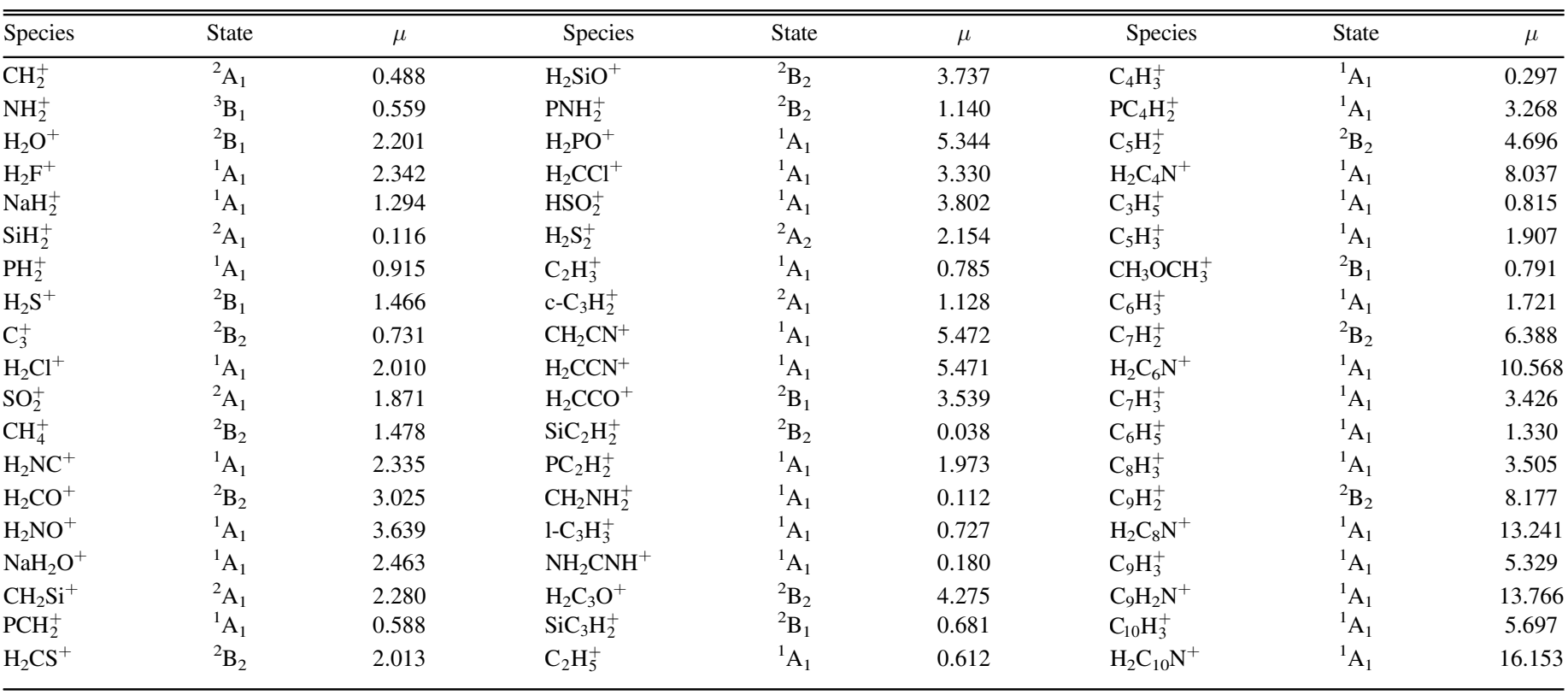

Note. $\mu$ is the electric dipole moment in Debye units.

Table 5

Predicted Equilibrium Properties of Planar Polyatomic Species without $\mathrm{C}_{2 v}$ Symmetry

\begin{tabular}{|c|c|c|c|c|c|c|c|}
\hline Species & State & Symmetry & $\mu$ & Species & State & Symmetry & $\mu$ \\
\hline $\mathrm{H}_{3}^{+}$ & ${ }^{1} \mathrm{~A}_{1}{ }^{\prime}$ & $\mathrm{D}_{3 h}$ & 0.000 & $\mathrm{HSiO}_{2}^{+}$ & ${ }^{1} A^{\prime}$ & $\mathrm{C}_{s}$ & 5.494 \\
\hline $\mathrm{HNO}^{+}$ & ${ }^{2} \mathrm{~A}^{\prime}$ & $\mathrm{C}_{s}$ & 2.908 & $\mathrm{H}_{2} \mathrm{COH}^{+}$ & ${ }^{1} A^{\prime}$ & $\mathrm{C}_{s}$ & 2.352 \\
\hline $\mathrm{HO}_{2}^{+}$ & ${ }^{3} \mathrm{~A}$ & $\mathrm{C}_{s}$ & 2.170 & $1-\mathrm{C}_{3} \mathrm{H}_{2}^{+}$ & ${ }^{2} A^{\prime}$ & $\mathrm{C}_{s}$ & 3.107 \\
\hline $\mathrm{HNS}^{+}$ & ${ }^{2} \mathrm{~A}^{\prime}$ & $\mathrm{C}_{s}$ & 1.439 & $\mathrm{HCOOH}^{+}$ & ${ }^{2} A^{\prime}$ & $\mathrm{C}_{s}$ & 0.432 \\
\hline $\mathrm{C}_{2} \mathrm{O}^{+}$ & ${ }^{2} \mathrm{~A}$ & $\mathrm{C}_{s}$ & 1.733 & $\mathrm{H}_{3} \mathrm{SiO}^{+}$ & ${ }^{1} A^{\prime}$ & $\mathrm{C}_{s}$ & 2.278 \\
\hline $\mathrm{HPO}^{+}$ & ${ }^{2} A^{\prime}$ & $\mathrm{C}_{s}$ & 2.962 & $\mathrm{PNH}_{3}^{+}$ & ${ }^{1} A^{\prime}$ & $\mathrm{C}_{s}$ & 3.093 \\
\hline $\mathrm{HSO}^{+}$ & ${ }^{1} \mathrm{~A}^{\prime}$ & $\mathrm{C}_{s}$ & 3.336 & c- $\mathrm{C}_{3} \mathrm{H}_{3}^{+}$ & ${ }^{1} \mathrm{~A}_{1}$ ' & $\mathrm{D}_{3 h}$ & 0.000 \\
\hline $\mathrm{CCP}^{+}$ & ${ }^{1} \mathrm{~A}^{\prime}$ & $\mathrm{C}_{s}$ & 2.381 & $\mathrm{SiC}_{2} \mathrm{H}_{3}^{+}$ & ${ }^{1} A^{\prime}$ & $\mathrm{C}_{s}$ & 0.224 \\
\hline $\mathrm{HS}_{2}^{+}$ & ${ }^{1} \mathrm{~A}^{\prime}$ & $\mathrm{C}_{s}$ & 1.496 & $\mathrm{NH}_{2} \mathrm{CH}_{2} \mathrm{O}^{+}$ & ${ }^{1} A^{\prime}$ & $\mathrm{C}_{s}$ & 2.190 \\
\hline $\mathrm{CH}_{3}^{+}$ & ${ }^{1} \mathrm{~A}_{1}{ }^{\prime}$ & $\mathrm{D}_{3 h}$ & 0.000 & $\mathrm{C}_{3} \mathrm{H}_{3} \mathrm{~N}^{+}$ & ${ }^{2} \mathrm{~A}^{\prime \prime}$ & $\mathrm{C}_{s}$ & 6.293 \\
\hline $\mathrm{NH}_{3}^{+}$ & ${ }^{2} \mathrm{~A}_{2} "$ & $\mathrm{D}_{3 h}$ & 0.000 & c- $\mathrm{C}_{3} \mathrm{H}_{2} \mathrm{OH}^{+}$ & ${ }^{1} A^{\prime}$ & $\mathrm{C}_{s}$ & 2.492 \\
\hline $\mathrm{SiH}_{3}^{+}$ & ${ }^{1} \mathrm{~A}_{1}{ }^{\prime}$ & $\mathrm{D}_{3 h}$ & 0.000 & $\mathrm{HCCCHOH}^{+}$ & ${ }^{1} A^{\prime}$ & $\mathrm{C}_{s}$ & 1.234 \\
\hline $\mathrm{HC}_{2} \mathrm{~N}^{+}$ & ${ }^{2} A^{\prime}$ & $\mathrm{C}_{s}$ & 5.002 & $\mathrm{C}_{2} \mathrm{H}_{3} \mathrm{CO}^{+}$ & ${ }^{1} A^{\prime}$ & $\mathrm{C}_{s}$ & 2.743 \\
\hline $\mathrm{C}_{2} \mathrm{HO}^{+}$ & ${ }^{1} \mathrm{~A}^{\prime}$ & $\mathrm{C}_{s}$ & 3.384 & $\mathrm{C}_{6} \mathrm{~N}^{+}$ & ${ }^{1} A^{\prime}$ & $\mathrm{C}_{s}$ & 5.324 \\
\hline $\mathrm{HNCO}^{+}$ & ${ }^{2} \mathrm{~A} "$ & $\mathrm{C}_{s}$ & 3.446 & $\mathrm{C}_{4} \mathrm{H}_{4}^{+}$ & ${ }^{2} A^{\prime \prime}$ & $\mathrm{C}_{s}$ & 1.078 \\
\hline $\mathrm{HOCO}^{+}$ & ${ }^{1} A^{\prime}$ & $\mathrm{C}_{s}$ & 3.446 & $\mathrm{C}_{3} \mathrm{H}_{3} \mathrm{NH}^{+}$ & ${ }^{1} A^{\prime}$ & $\mathrm{C}_{s}$ & 1.707 \\
\hline $\mathrm{HN}_{2} \mathrm{O}^{+}$ & ${ }^{1} A^{\prime}$ & $\mathrm{C}_{s}$ & 3.706 & $\mathrm{COOCH}_{4}^{+}$ & ${ }^{2} A^{\prime}$ & $\mathrm{C}_{s}$ & 3.196 \\
\hline $\mathrm{C}_{3} \mathrm{~N}^{+}$ & ${ }^{3} \mathrm{~A} "$ & $\mathrm{C}_{s}$ & 2.921 & $\mathrm{C}_{5} \mathrm{H}_{5}^{+}$ & ${ }^{1} A^{\prime}$ & $\mathrm{C}_{s}$ & 1.219 \\
\hline $\mathrm{SiNCH}^{+}$ & ${ }^{2} A^{\prime}$ & $\mathrm{C}_{s}$ & 2.156 & $\mathrm{C}_{6} \mathrm{H}_{4}^{+}$ & ${ }^{2} \mathrm{~A}_{u}$ & $\mathrm{C}_{2 h}$ & 0.000 \\
\hline $\mathrm{HOCS}^{+}$ & ${ }^{1} \mathrm{~A}^{\prime}$ & $\mathrm{C}_{s}$ & 2.274 & & & & \\
\hline
\end{tabular}

Note. $\mu$ is the electric dipole moment in Debye units.

is $0.308 \mathrm{eV}$. The difference between these two quantities is that in vertical IE (experimental) the geometry of the ionized molecule is not allowed to relax in its correponding PES, while in adiabatic IE (computed) the energy of the ion is taken from the minimum of its PES. Therefore, adiabatic IE are expected to be always lower with respect to vertical IE, if calculated with the same methodology.

Connectivity change between neutral and cationic partners - Using the tools described in the Section 2.4, the atomic connectivity check was performed on the cations reported in
Table 7 and compared to the corresponding neutral species. The only cations that change their connectivity after the ionization and the subsequent geometric optimization are: $\mathrm{C}_{3}^{+}$, $\mathrm{C}_{2} \mathrm{H}_{5}^{+}$, and $\mathrm{C}_{4} \mathrm{H}_{5}^{+}$, as shown in Figure 4. For the $\mathrm{C}_{3}^{+}$this rearrangement explains the large difference $(\sim 1.4 \mathrm{eV})$ between our computed IE and the experimental vertical IE value.

This result corroborates our adopted methodology (Section 2), because it also demonstrates that the assumption in the astrochemical networks that many cations share the same connectivity as their neutral counterparts is respected. 


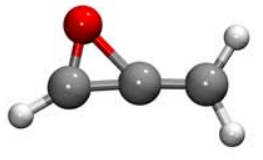

(a) $\mathrm{C}_{2} \mathrm{H}_{3} \mathrm{CO}^{+}$

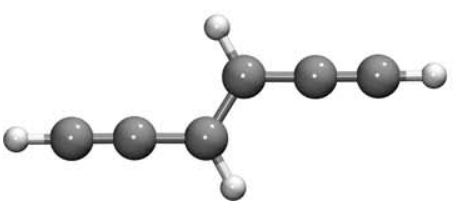

(e) $\mathrm{C}_{6} \mathrm{H}_{4}^{+}$

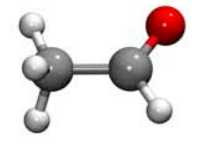

(b) $\mathrm{C}_{2} \mathrm{H}_{4} \mathrm{O}^{+}$

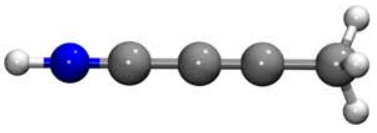

(f) $\mathrm{H}_{3} \mathrm{C}_{4} \mathrm{NH}^{+}$

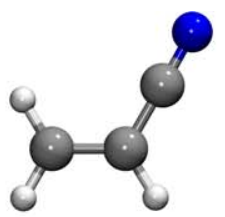

(c) $\mathrm{C}_{3} \mathrm{H}_{3} \mathrm{~N}^{+}$

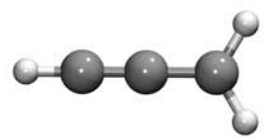

(g) $\mathrm{l}_{-} \mathrm{C}_{3} \mathrm{H}_{3}^{+}$

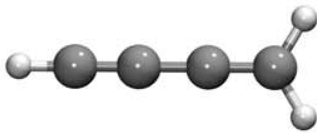

(d) $\mathrm{C}_{4} \mathrm{H}_{3}^{+}$

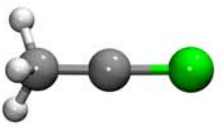

(h) $\mathrm{PC}_{2} \mathrm{H}_{3}^{+}$

Figure 3. M06-2X/cc-pVTZ optimized structures of a sample of the cations studied in the present work. The structure of all the 262 cations is available online as data behind the figure and at the ACO Cations Database website (aco-itn.oapd.inaf.it/aco-public-datasets/theoretical-chemistry-calculations/cations-database).

(The data used to create this figure are available.)

Table 6

Predicted Equilibrium Properties of Nonplanar Polyatomic Species

\begin{tabular}{|c|c|c|c|c|c|c|c|}
\hline$\overline{\text { Species }}$ & State & Symmetry & $\mu$ & Species & State & Symmetry & $\overline{\mu \mu}$ \\
\hline$\overline{\mathrm{H}_{3} \mathrm{O}^{+}}$ & ${ }^{1} \mathrm{~A}_{1}$ & $\mathrm{C}_{3 v}$ & 1.414 & $\mathrm{CH}_{3} \mathrm{NH}_{3}^{+}$ & ${ }^{1} \mathrm{~A}_{1}$ & $\mathrm{C}_{3 v}$ & 2.166 \\
\hline $\mathrm{PH}_{3}^{+}$ & ${ }^{2} \mathrm{~A}_{1}$ & $\mathrm{C}_{3 v}$ & 0.359 & $\mathrm{CH}_{3} \mathrm{CHOH}^{+}$ & ${ }^{1} \mathrm{~A}$ ' & $\mathrm{C}_{s}$ & 2.569 \\
\hline $\mathrm{H}_{3} \mathrm{~S}^{+}$ & ${ }^{1} \mathrm{~A}_{1}$ & $\mathrm{C}_{3 v}$ & 1.554 & $\mathrm{H}_{3} \mathrm{C}_{4} \mathrm{~N}^{+}$ & ${ }^{2} \mathrm{~A}^{\prime}$ & $\mathrm{C}_{s}$ & 5.327 \\
\hline $\mathrm{NH}_{4}^{+}$ & ${ }^{1} \mathrm{~A}_{1}$ & $\mathrm{~T}_{d}$ & 0.000 & $\mathrm{C}_{2} \mathrm{H}_{5} \mathrm{OH}^{+}$ & ${ }^{2} \mathrm{~A}$ & $\mathrm{C}_{1}$ & 2.083 \\
\hline $\mathrm{SiH}_{4}^{+}$ & ${ }^{2} \mathrm{~A}^{\prime}$ & $\mathrm{C}_{s}$ & 1.222 & $\mathrm{C}_{4} \mathrm{H}_{5}^{+}$ & ${ }^{1} \mathrm{~A}$, & $\mathrm{C}_{s}$ & 0.860 \\
\hline $\mathrm{SiCH}_{3}^{+}$ & ${ }^{1} \mathrm{~A}_{1}$ & $\mathrm{C}_{3 v}$ & 0.750 & $\mathrm{H}_{5} \mathrm{C}_{2} \mathrm{O}_{2}^{+}$ & ${ }^{1} \mathrm{~A}^{\prime}$ & $\mathrm{C}_{s}$ & 0.972 \\
\hline $\mathrm{H}_{3} \mathrm{CS}^{+}$ & ${ }^{3} \mathrm{~A}_{1}$ & $\mathrm{C}_{3 v}$ & 0.559 & $\mathrm{C}_{5} \mathrm{H}_{4}^{+}$ & ${ }^{2} \mathrm{~B}_{3}$ & $D_{2}$ & 0.000 \\
\hline $\mathrm{PCH}_{3}^{+}$ & ${ }^{2} \mathrm{~A} "$ & $\mathrm{C}_{s}$ & 0.249 & $\mathrm{H}_{3} \mathrm{C}_{4} \mathrm{NH}^{+}$ & ${ }^{1} \mathrm{~A}_{1}$ & $\mathrm{C}_{3 v}$ & 2.635 \\
\hline $\mathrm{H}_{3} \mathrm{~S}_{2}^{+}$ & ${ }^{1} \mathrm{~A}$ & $\mathrm{C}_{1}$ & 2.292 & $\mathrm{HCOCH}_{2} \mathrm{OH}_{2}^{+}$ & ${ }^{1} \mathrm{~A}^{\prime}$ & $\mathrm{C}_{s}$ & 2.474 \\
\hline $\mathrm{CH}_{5}^{+}$ & ${ }^{1} \mathrm{~A}^{\prime}$ & $\mathrm{C}_{s}$ & 1.630 & $\mathrm{C}_{5} \mathrm{H}_{3} \mathrm{~N}^{+}$ & ${ }^{2} \mathrm{~A} "$ & $\mathrm{C}_{s}$ & 6.270 \\
\hline $\mathrm{C}_{2} \mathrm{H}_{4}^{+}$ & ${ }^{2} \mathrm{~B}_{3}$ & $\mathrm{D}_{2}$ & 0.000 & $\mathrm{C}_{2} \mathrm{H}_{5} \mathrm{OH}_{2}^{+}$ & ${ }^{1} \mathrm{~A}$ & $\mathrm{C}_{1}$ & 3.312 \\
\hline $\mathrm{CH}_{3} \mathrm{OH}^{+}$ & ${ }^{2} \mathrm{~A} "$ & $\mathrm{C}_{s}$ & 1.394 & $\mathrm{CH}_{3} \mathrm{OCH}_{4}^{+}$ & ${ }^{1} \mathrm{~A}$ & $\mathrm{C}_{s}$ & 1.177 \\
\hline $\mathrm{CH}_{3} \mathrm{CO}^{+}$ & ${ }^{1} \mathrm{~A}_{1}$ & $\mathrm{C}_{3 v}$ & 2.977 & $\mathrm{C}_{2} \mathrm{H}_{6} \mathrm{CO}^{+}$ & ${ }^{2} \mathrm{~B}$ & $\mathrm{C}_{2}$ & 1.567 \\
\hline $\mathrm{SiH}_{5}^{+}$ & ${ }^{1} \mathrm{~A}^{\prime}$ & $\mathrm{C}_{s}$ & 1.284 & $\mathrm{C}_{5} \mathrm{H}_{4} \mathrm{~N}^{+}$ & ${ }^{1} \mathrm{~A}$ & $\mathrm{C}_{1}$ & 5.401 \\
\hline $\mathrm{SiCH}_{4}^{+}$ & ${ }^{2} \mathrm{~A}^{\prime}$ & $\mathrm{C}_{s}$ & 1.224 & $\mathrm{C}_{4} \mathrm{H}_{7}^{+}$ & ${ }^{1} \mathrm{~A}$, & $\mathrm{C}_{s}$ & 1.480 \\
\hline $\mathrm{PCH}_{4}^{+}$ & ${ }^{3} \mathrm{~A} "$ & $\mathrm{C}_{s}$ & 0.858 & $\mathrm{C}_{3} \mathrm{H}_{6} \mathrm{OH}^{+}$ & ${ }^{1} \mathrm{~A}$ & $\mathrm{C}_{1}$ & 1.636 \\
\hline $\mathrm{PC}_{2} \mathrm{H}_{3}^{+}$ & ${ }^{2} \mathrm{~A}^{\prime}$ & $\mathrm{C}_{s}$ & 0.375 & $\mathrm{C}_{7} \mathrm{H}_{4}^{+}$ & ${ }^{2} \mathrm{~B}_{2}$ & $D_{2}$ & 0.000 \\
\hline $\mathrm{CH}_{3} \mathrm{O}_{2}^{+}$ & ${ }^{1} \mathrm{~A}$ & $\mathrm{C}_{1}$ & 4.737 & $\mathrm{H}_{3} \mathrm{C}_{6} \mathrm{NH}^{+}$ & ${ }^{1} \mathrm{~A}_{1}$ & $\mathrm{C}_{3 v}$ & 4.646 \\
\hline $\mathrm{CH}_{3} \mathrm{CN}^{+}$ & ${ }^{2} A^{\prime}$ & $\mathrm{C}_{s}$ & 2.604 & $\mathrm{H}_{3} \mathrm{C}_{7} \mathrm{~N}^{+}$ & ${ }^{2} \mathrm{~A}$ & $\mathrm{C}_{1}$ & 5.120 \\
\hline $\mathrm{CH}_{3} \mathrm{NH}_{2}^{+}$ & ${ }^{2} \mathrm{~A}^{\prime}$ & $\mathrm{C}_{s}$ & 1.974 & $\mathrm{C}_{7} \mathrm{H}_{5}^{+}$ & ${ }^{1} \mathrm{~A}$, & $\mathrm{C}_{s}$ & 2.172 \\
\hline $\mathrm{CH}_{3} \mathrm{OH}_{2}^{+}$ & ${ }^{1} \mathrm{~A}^{\prime}$ & $\mathrm{C}_{s}$ & 1.835 & $\mathrm{C}_{8} \mathrm{H}_{4}^{+}$ & ${ }^{2} \mathrm{~B}_{2}$ & $D_{2}$ & 0.000 \\
\hline $\mathrm{C}_{3} \mathrm{H}_{4}^{+}$ & ${ }^{2} \mathrm{~B}_{2}$ & $\mathrm{D}_{2}$ & 0.000 & $\mathrm{C}_{6} \mathrm{H}_{7}^{+}$ & ${ }^{1} \mathrm{~A}^{\prime}$ & $\mathrm{C}_{s}$ & 0.780 \\
\hline $\mathrm{CH}_{3} \mathrm{CNH}^{+}$ & ${ }^{1} \mathrm{~A}_{1}$ & $\mathrm{C}_{3 v}$ & 1.036 & $\mathrm{C}_{8} \mathrm{H}_{5}^{+}$ & ${ }^{1} \mathrm{~A}$, & $\mathrm{C}_{s}$ & 3.950 \\
\hline $\mathrm{C}_{2} \mathrm{H}_{4} \mathrm{O}^{+}$ & ${ }^{2} \mathrm{~A}^{\prime}$ & $\mathrm{C}_{s}$ & 2.056 & $\mathrm{C}_{9} \mathrm{H}_{4}^{+}$ & ${ }^{2} \mathrm{~A}$ & $\mathrm{C}_{1}$ & 0.391 \\
\hline $\mathrm{PC}_{2} \mathrm{H}_{4}^{+}$ & ${ }^{1} \mathrm{~A}^{\prime}$ & $\mathrm{C}_{s}$ & 0.573 & $\mathrm{C}_{8} \mathrm{H}_{4} \mathrm{~N}^{+}$ & ${ }^{1} \mathrm{~A}_{1}$ & $\mathrm{C}_{3 v}$ & 7.166 \\
\hline $\mathrm{C}_{2} \mathrm{H}_{6}^{+}$ & ${ }^{2} \mathrm{~A}_{g}$ & $\mathrm{C}_{2 h}$ & 0.000 & $\mathrm{C}_{9} \mathrm{H}_{5}^{+}$ & ${ }^{1} \mathrm{~A}$ & $\mathrm{C}_{s}$ & 3.960 \\
\hline
\end{tabular}

Note. $\mu$ is the electric dipole moment in Debye units.

\section{Discussion}

As explained in the Introduction, having the physicochemical properties of the species involved in the astrochemical networks is a first basic step toward achieving accurate and reliable modeling. In this article, we complemented the work by Woon \& Herbst (2009) on neutral species adding the physicochemical properties of the totality of cations present in the KIDA network ${ }^{7}$. In the following, we discuss a first, immediate application of the combined neutral plus cations data sets: how the reaction energy, derived from the neutral and cations data sets, could be used to identify reactions that cannot happen in the conditions in the interstellar medium.

This example concerns two reactions, reported in Table 8, which involve $\mathrm{SiS}^{+}$. This cation has been postulated to be a precursor leading to $\mathrm{SiS}$, a species observed in star-forming regions and associated with the molecular shocks of young forming protostars (Tercero et al. 2011; Podio et al. 2017). In 
Table 7

Adiabatic Ionization Energy of the Corresponding Neutral Species

\begin{tabular}{|c|c|c|c|c|c|c|c|c|c|c|c|}
\hline Species & $\mathrm{eV}$ & $\mathrm{eV}_{(\exp )}$ & Species & $\mathrm{eV}$ & $\mathrm{eV}_{(\mathrm{exp})}$ & Species & $\mathrm{eV}$ & $\mathrm{eV}_{(\exp )}$ & Species & $\mathrm{eV}$ & $\mathrm{eV}_{(\exp )}$ \\
\hline$\overline{\mathrm{H}_{2}^{+}}$ & 15.528 & $15.426^{\mathrm{a}}$ & $\mathrm{HNC}^{+}$ & 11.961 & $12.500^{\text {ai }}$ & $\mathrm{C}_{3} \mathrm{~N}^{+}$ & 11.816 & & $\mathrm{C}_{6} \mathrm{H}^{+}$ & 9.336 & \\
\hline $\mathrm{CH}^{+}$ & 10.562 & $10.640^{\mathrm{b}}$ & $\mathrm{HCO}^{+}$ & 8.037 & $8.140^{\mathrm{al}}$ & $\mathrm{C}_{3} \mathrm{O}^{+}$ & 10.772 & & $\mathrm{HC}_{5} \mathrm{~N}^{+}$ & 10.558 & \\
\hline $\mathrm{NH}^{+}$ & 13.420 & $13.490^{c}$ & $\mathrm{SiH}_{2}^{+}$ & 9.101 & & $\mathrm{SiC}_{2} \mathrm{H}^{+}$ & 7.289 & & $\mathrm{C}_{7}^{+}$ & 10.293 & \\
\hline $\mathrm{OH}^{+}$ & 12.899 & $13.017^{\mathrm{d}}$ & $\mathrm{HNO}^{+}$ & 10.165 & $10.100^{\mathrm{am}}$ & $\mathrm{C}_{3} \mathrm{~S}^{+}$ & 10.188 & & $\mathrm{C}_{6} \mathrm{~N}^{+}$ & 8.866 & \\
\hline $\mathrm{HF}^{+}$ & 15.998 & $15.980^{\mathrm{e}}$ & $\mathrm{PH}_{2}^{+}$ & 9.826 & $9.824^{\mathrm{m}}$ & $\mathrm{CH}_{4}^{+}$ & 12.749 & $12.610^{\mathrm{bh}}$ & $\mathrm{C}_{2} \mathrm{H}_{6}^{+}$ & 11.619 & $11.570^{\mathrm{bw}}$ \\
\hline $\mathrm{C}_{2}^{+}$ & 11.728 & $11.920^{\mathrm{f}}$ & $\mathrm{HO}_{2}^{+}$ & 11.306 & $11.350^{\mathrm{an}}$ & $\mathrm{C}_{2} \mathrm{H}_{3}^{+}$ & 8.678 & $8.250^{\mathrm{bi}}$ & $\mathrm{C}_{3} \mathrm{H}_{5}^{+}$ & 8.029 & \\
\hline $\mathrm{CN}^{+}$ & 13.715 & $14.170^{\mathrm{b}}$ & $\mathrm{H}_{2} \mathrm{~S}^{+}$ & 10.451 & $10.453^{\mathrm{ao}}$ & $\mathrm{SiH}_{4}^{+}$ & 11.050 & $11.200^{\mathrm{bl}}$ & $\mathrm{CH}_{3} \mathrm{CHOH}^{+}$ & 6.662 & \\
\hline $\mathrm{CO}^{+}$ & 13.959 & $14.014^{\mathrm{g}}$ & $\mathrm{C}_{3}^{+}$ & 11.606 & $11.610^{\mathrm{ap}}$ & $\mathrm{c}-\mathrm{C}_{3} \mathrm{H}_{2}^{+}$ & 9.118 & $9.150^{\mathrm{bm}}$ & $\mathrm{C}_{5} \mathrm{H}_{3}^{+}$ & 8.124 & \\
\hline $\mathrm{N}_{2}^{+}$ & 15.533 & $15.581^{\mathrm{h}}$ & $\mathrm{C}_{2} \mathrm{~N}^{+}$ & 10.730 & $12.000^{\mathrm{aq}}$ & $\mathrm{CH}_{2} \mathrm{CN}^{+}$ & 10.211 & $10.300^{\mathrm{bn}}$ & $\mathrm{C}_{6} \mathrm{H}_{2}^{+}$ & 9.477 & \\
\hline $\mathrm{SiH}^{+}$ & 7.900 & $7.890^{\mathrm{i}}$ & $\mathrm{C}_{2} \mathrm{O}^{+}$ & 10.912 & & $\mathrm{H}_{2} \mathrm{CCO}^{+}$ & 9.538 & $9.614^{\mathrm{bo}}$ & $\mathrm{C}_{7} \mathrm{H}^{+}$ & 8.062 & \\
\hline $\mathrm{NO}^{+}$ & 9.191 & $9.264^{1}$ & $\mathrm{NCO}^{+}$ & 11.620 & $11.759^{\mathrm{ar}}$ & $\mathrm{SiCH}_{3}^{+}$ & 7.066 & & $\mathrm{HC}_{6} \mathrm{~N}^{+}$ & 2.494 & \\
\hline $\mathrm{PH}^{+}$ & 10.160 & $10.149^{\mathrm{m}}$ & $\mathrm{HNSi}^{+}$ & 11.718 & & $\mathrm{HCOOH}^{+}$ & 11.282 & $11.310^{\mathrm{bp}}$ & $\mathrm{C}_{8}^{+}$ & 11.376 & \\
\hline $\mathrm{O}_{2}^{+}$ & 12.053 & $12.070^{\mathrm{n}}$ & $\mathrm{HCP}^{+}$ & 10.786 & $10.790^{\text {as }}$ & $\mathrm{C}_{4} \mathrm{H}^{+}$ & 10.057 & & $\mathrm{C}_{7} \mathrm{~N}^{+}$ & 9.659 & \\
\hline $\mathrm{HS}^{+}$ & 10.310 & $10.421^{\circ}$ & $\mathrm{CO}_{2}^{+}$ & 13.765 & $13.778^{\text {at }}$ & $\mathrm{HC}_{3} \mathrm{~N}^{+}$ & 11.620 & & $\mathrm{CH}_{3} \mathrm{OCH}_{3}^{+}$ & 10.004 & $10.025^{\mathrm{bz}}$ \\
\hline $\mathrm{HCl}^{+}$ & 12.716 & $12.790^{\mathrm{p}}$ & $\mathrm{HCS}^{+}$ & 7.588 & $7.412^{\mathrm{au}}$ & $\mathrm{C}_{5}^{+}$ & 11.116 & $12.300^{\mathrm{bq}}$ & $\mathrm{C}_{4} \mathrm{H}_{5}^{+}$ & 7.066 & \\
\hline $\mathrm{SiC}^{+}$ & 8.853 & $9.000^{\mathrm{q}}$ & $\mathrm{NO}_{2}^{+}$ & 9.458 & $9.600^{\mathrm{av}}$ & $\mathrm{C}_{4} \mathrm{~N}^{+}$ & 9.514 & & $\mathrm{C}_{8} \mathrm{H}^{+}$ & 8.836 & \\
\hline $\mathrm{SiN}^{+}$ & 10.342 & & $\mathrm{HPO}^{+}$ & 10.548 & & $\mathrm{SiC}_{3} \mathrm{H}^{+}$ & 8.181 & & $\mathrm{HC}_{7} \mathrm{~N}^{+}$ & 9.825 & \\
\hline $\mathrm{CP}^{+}$ & 10.914 & $10.500^{\mathrm{r}}$ & $\mathrm{SiNC}^{+}$ & 7.851 & & $\mathrm{SiC}_{4}^{+}$ & 10.099 & & $\mathrm{C}_{9}^{+}$ & 3.734 & \\
\hline $\mathrm{CS}^{+}$ & 11.381 & $11.330^{\mathrm{s}}$ & $\mathrm{C}_{2} \mathrm{~S}^{+}$ & 10.186 & & $\mathrm{C}_{4} \mathrm{P}^{+}$ & 8.447 & & $\mathrm{C}_{8} \mathrm{~N}^{+}$ & 8.430 & \\
\hline $\mathrm{PN}^{+}$ & 11.933 & $11.880^{t}$ & $\mathrm{OCS}^{+}$ & 11.189 & $11.185^{\text {aw }}$ & $\mathrm{C}_{4} \mathrm{~S}^{+}$ & 9.199 & & $\mathrm{C}_{2} \mathrm{H}_{6} \mathrm{CO}^{+}$ & 9.675 & $9.700^{\mathrm{ca}}$ \\
\hline $\mathrm{NS}^{+}$ & 8.916 & $8.870^{\mathrm{u}}$ & $\mathrm{HSiS}^{+}$ & 8.260 & & $\mathrm{C}_{2} \mathrm{H}_{4}^{+}$ & 10.485 & $10.510^{\mathrm{br}}$ & $\mathrm{C}_{5} \mathrm{H}_{5}^{+}$ & 7.718 & \\
\hline $\mathrm{PO}^{+}$ & 8.476 & $8.390^{\mathrm{v}}$ & $\mathrm{SO}_{2}^{+}$ & 12.680 & $12.500^{\mathrm{az}}$ & $\mathrm{CH}_{3} \mathrm{OH}^{+}$ & 10.928 & $10.850^{\mathrm{bs}}$ & $\mathrm{C}_{6} \mathrm{H}_{4}^{+}$ & 8.993 & \\
\hline $\mathrm{CCl}^{+}$ & 8.834 & $8.900^{w}$ & $\mathrm{HS}_{2}^{+}$ & 9.376 & & $\mathrm{CH}_{3} \mathrm{CN}^{+}$ & 12.246 & $12.201^{\mathrm{bt}}$ & $\mathrm{C}_{8} \mathrm{H}_{2}^{+}$ & 8.978 & \\
\hline $\mathrm{SiO}^{+}$ & 11.521 & $11.300^{\mathrm{z}}$ & $\mathrm{CH}_{3}^{+}$ & 9.736 & $9.843^{\text {ba }}$ & $\mathrm{C}_{4} \mathrm{H}_{2}^{+}$ & 10.131 & & $\mathrm{C}_{9} \mathrm{H}^{+}$ & 7.742 & \\
\hline $\mathrm{SO}^{+}$ & 10.428 & $10.294^{\mathrm{aa}}$ & $\mathrm{NH}_{3}^{+}$ & 10.148 & $10.020^{\mathrm{bb}}$ & $\mathrm{C}_{5} \mathrm{H}^{+}$ & 8.358 & & $\mathrm{HC}_{8} \mathrm{~N}^{+}$ & 8.387 & \\
\hline $\mathrm{SiS}^{+}$ & 10.526 & $10.530^{\mathrm{ab}}$ & $\mathrm{C}_{2} \mathrm{H}_{2}^{+}$ & 11.330 & $11.410^{\mathrm{bc}}$ & $\mathrm{HC}_{4} \mathrm{~N}^{+}$ & 9.425 & & $\mathrm{C}_{10}^{+}$ & 10.647 & \\
\hline $\mathrm{S}_{2}^{+}$ & 9.503 & $9.400^{\mathrm{ac}}$ & $\mathrm{H}_{2} \mathrm{CO}^{+}$ & 10.900 & $10.880^{\mathrm{bd}}$ & $\mathrm{C}_{6}^{+}$ & 12.441 & & $\mathrm{C}_{9} \mathrm{~N}^{+}$ & 9.168 & \\
\hline $\mathrm{CH}_{2}^{+}$ & 10.338 & $10.350^{\mathrm{ad}}$ & $\mathrm{SiH}_{3}^{+}$ & 8.109 & $8.170^{\text {be }}$ & $\mathrm{C}_{5} \mathrm{~N}^{+}$ & 10.705 & & $\mathrm{C}_{10} \mathrm{H}^{+}$ & 8.510 & \\
\hline $\mathrm{NH}_{2}^{+}$ & 11.131 & $10.780^{\text {ae }}$ & $\mathrm{HNCO}^{+}$ & 11.557 & $11.595^{\mathrm{bf}}$ & $\mathrm{SiC}_{4} \mathrm{H}^{+}$ & 7.082 & & $\mathrm{C}_{11}^{+}$ & 8.984 & \\
\hline $\mathrm{H}_{2} \mathrm{O}^{+}$ & 12.574 & $12.650^{\mathrm{af}}$ & $\mathrm{H}_{2} \mathrm{CS}^{+}$ & 9.411 & $9.376^{\mathrm{bg}}$ & $\mathrm{C}_{2} \mathrm{H}_{5}^{+}$ & 8.063 & $8.117^{\text {bu }}$ & $\mathrm{C}_{10} \mathrm{~N}^{+}$ & 8.026 & \\
\hline $\mathrm{C}_{2} \mathrm{H}^{+}$ & 11.267 & $11.610^{\mathrm{ag}}$ & $\mathrm{H}_{2} \mathrm{SiO}^{+}$ & 10.674 & & $\mathrm{CH}_{3} \mathrm{NH}_{2}^{+}$ & 9.061 & $8.900^{\text {bv }}$ & $\mathrm{C}_{10} \mathrm{H}_{2}^{+}$ & 8.722 & \\
\hline $\mathrm{HCN}^{+}$ & 13.543 & $13.590^{\mathrm{ah}}$ & $\mathrm{C}_{4}^{+}$ & 10.734 & & $\mathrm{C}_{4} \mathrm{H}_{3}^{+}$ & 7.960 & & $\mathrm{HC}_{10} \mathrm{~N}^{+}$ & 8.094 & \\
\hline
\end{tabular}

Notes. Adiabatic ionization energy $(\mathrm{eV})$ without $\mathrm{ZPE}$ correction, compared with the vertical experimental ionization energy $\left(\mathrm{eV}_{(\exp )}\right)$.

${ }^{\mathrm{a}}$ (Shiner et al. 1993). ${ }^{\mathrm{b}}$ (Huber 2013). ${ }^{\mathrm{c}}$ (Dyke et al. 1980). ${ }^{\mathrm{d}}$ (Wiedmann et al. 1992). ${ }^{\mathrm{e}}$ (Tiedemann et al. 1979). ${ }^{\mathrm{f}}$ (Plessis \& Marmet 1987). ${ }^{\mathrm{g}}$ (Erman et al. 1993). ${ }^{\mathrm{h}}$ (Trickl et al. 1989). ${ }^{\mathrm{i}}$ (Boo \& Armentrout 1987). ${ }^{\mathrm{l}}$ (Reiser et al. 1988). ${ }^{\mathrm{m}}$ (Berkowitz \& Cho 1989). ${ }^{\mathrm{n}}$ (Tonkyn et al. 1989). ${ }^{\mathrm{o}}$ (Milan et al. 1996). ${ }^{\mathrm{p}}$ (Wang et al. 1984). ${ }^{\mathrm{q}}$ (Verhaegen et al. 1964). ${ }^{\mathrm{r}}$ (Smoes et al. 1971). ${ }^{\mathrm{s}}$ (Drowart et al. 1978). ${ }^{\mathrm{t}}$ (Bulgin et al. 1977). ${ }^{\mathrm{u}}$ (Dyke et al. 1977). ${ }^{\mathrm{v}}$ (Dyke et al. 1982). ${ }^{\mathrm{w}}$ (Hepburn et al. 1982). ${ }^{\mathrm{z}}$ (Nakasgawa et al. 1981). ${ }^{\text {aa }}$ (Norwood \& Ng 1989a). ${ }^{\text {ab }}$ (Cockett et al. 1989). ${ }^{\text {ac }}$ (Bender et al. 1988). ${ }^{\text {ad }}$ (Reineke \& Strein 1976). ${ }^{\text {ae }}$ (Qi et al. 1995). ${ }^{\text {af }}$ (Snow \& Thomas 1990). ${ }^{\text {ag }}$ (Norwood \& Ng 1989b). ${ }^{\text {ah }}$ (Dibeler \& Liston 1968). ${ }^{\text {ai }}$ (Bieri \& Jonsson 1978). ${ }^{\text {al }}$ (Dyke 1987). ${ }^{\text {am }}$ (Lias et al. 1988). ${ }^{\text {an }}$ (Tang et al. 2020). ${ }^{\text {ao }}$ (Walters \& Blais 1984). ${ }^{\text {ap }}$ Rohlfing et al. (1984); only the upper limit value was reported. ${ }^{\text {aq }}$ (Lias et al. 1988). ${ }^{\text {ar }}$ (Dyke 1987). ${ }^{\text {as }}$ (Frost et al. 1973). ${ }^{\text {at }}$ (Wang et al. 1988). ${ }^{\text {au }}$ (Ruscic \& Berkowitz 1993). ${ }^{\text {av }}$ (Clemmer \& Armentrout 1992). ${ }^{\text {aw }}$ (Wang et al. 1988). ${ }^{\text {az }}$ (Snow \& Thomas 1990). ${ }^{\text {ba }}$ (Berkowitz et al. 1994). ${ }^{\text {bb }}$ (Qi et al. 1995). ${ }^{\text {bc }}$ (Plessis \& Marmet 1986). ${ }^{\text {bd }}$ (Ohno et al. 1995). ${ }^{\text {be }}$ (Nagano et al. 1993). ${ }^{\text {bf }}$ (Ruscic \& Berkowitz 1994). ${ }^{\text {bg }}$ (Ruscic \& Berkowitz 1993). ${ }^{\text {bh }}$ (Berkowitz et al. 1987). ${ }^{\text {bi }}$ (Blush \& Chen 1992). ${ }^{\text {bl }}$ (Shin et al. 1990). ${ }^{\text {bm }}$ (Clauberg et al. 1992). ${ }^{\text {bn }}$ (Holmes et al. 1993). ${ }^{\text {bo }}$ (Vogt et al. 1978). ${ }^{\text {bp }}$ (Traeger 1985). ${ }^{\text {bq }}$ (Ramanathan et al. 1993). ${ }^{\text {br }}$ (Ohno et al. 1995). ${ }^{\text {bs }}$ (Tao et al. 1992). ${ }^{\text {bt }}$ (Gochel-Dupuis et al. 1992). ${ }^{\text {bu }}$ (Ruscic et al. 1989). ${ }^{\text {bv }}$ (Aue \& Bowers 1979). ${ }^{\text {bw }}$ (Plessis \& Marmet 1987). ${ }^{\mathrm{bz}}$ (Butler et al. 1984). ${ }^{\mathrm{ca}}$ (Traeger et al. 1982).

The calculated adiabatic ionization energies for $\mathrm{C}_{4}, \mathrm{C}_{6}, \mathrm{C}_{8}$, and $\mathrm{C}_{10}$ have been computed with respect to the triplet state of these species and not to the singlet state, as reported in Woon \& Herbst (2009).

contrast to the competing S-bearing species $\mathrm{SiO}$, which is likely extracted from the shattered grains, $\mathrm{SiS}$ is though to be a product of gas-phase reactions (Podio et al. 2017). The KIDA database only lists one reaction forming $\mathrm{SiS}: \mathrm{HSiS}^{+}+\mathrm{e} \rightarrow \mathrm{SiS}$ $+\mathrm{H}$. In turn, according to KIDA, $\mathrm{HSiS}^{+}$is formed by the first reaction in Table 8: $\mathrm{H}_{2}+\mathrm{SiS}^{+} \rightarrow \mathrm{H}+\mathrm{HSiS}^{+}$. Our computations, coupled with those by Woon \& Herbst (2009), clearly show that the this reaction is highly endothermic $(\sim 104 \mathrm{~kJ}$ $\mathrm{mol}^{-1}$ ) and, consequently, rule out the formation of SiS by the recombination of $\mathrm{HSiS}^{+}$. Previous experimental work by Wlodek \& Bohme (1989) support our conclusion. Therefore, these two reactions (the formation of $\mathrm{SiS}$ from $\mathrm{HSiS}^{+}$) should be removed by the astrochemical reaction databases.

The second reaction in Table 8 involves the formation of $\mathrm{SiS}^{+}$, which would be the step before the above SiS reaction formation, according to the KIDA database (on the contrary, the UMIST database does not report the reaction). Also, in this case, our calculations show that the reaction is endothermic $\left(\sim 36 \mathrm{~kJ} \mathrm{~mol}^{-1}\right)$ and, therefore, should be removed from the database.

For the curious reader, other routes of $\mathrm{SiS}$ formation, involving neutral-neutral reactions, have been explored in the literature 

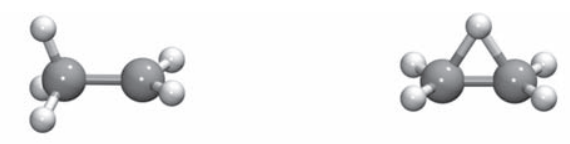

(a) $\mathrm{C}_{2} \mathrm{H}_{5}$ and $\mathrm{C}_{2} \mathrm{H}_{5}^{+}$
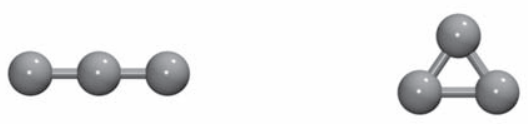

(b) $\mathrm{C}_{3}$ and $\mathrm{C}_{3}^{+}$
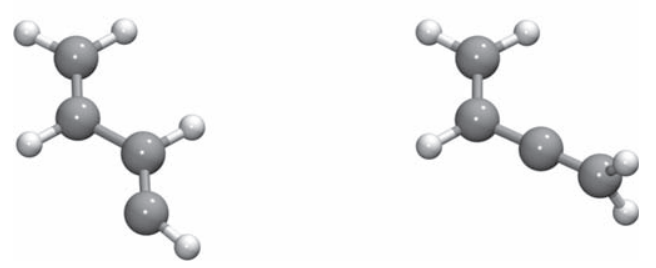

(c) $\mathrm{C}_{4} \mathrm{H}_{5}$ and $\mathrm{C}_{4} \mathrm{H}_{5}^{+}$

Figure 4. Connectivity change between cations and the neutral species.

Table 8

Two Examples of Endothermic Reactions Found in the Astrochemical Reaction Network for Molecular Clouds

\begin{tabular}{lc}
\hline \hline Reaction & $\Delta E\left[\mathrm{~kJ} \mathrm{~mol}^{-1}\right]$ \\
\hline $\mathrm{H}_{2}+\mathrm{SiS}^{+} \rightarrow \mathrm{H}+\mathrm{HSiS}^{+}$ & 103.6 \\
$\mathrm{SiS}+\mathrm{S}^{+} \rightarrow \mathrm{SiS}^{+}+\mathrm{S}$ & 36.1 \\
\hline
\end{tabular}

Note. The reaction energy (i.e., $\Delta E$, no ZPE correction) is computed with data from this work and Woon \& Herbst (2009).

since the work of Podio et al. (2017) and found to be plausible (Rosi et al. 2018; Zanchet et al. 2018; Rosi et al. 2019).

\section{Conclusions}

In this work, we present new ab initio calculations of the structure and energy of 262 cations, all appearing in the used astrochemical reaction network databases KIDA and UMIST. Our calculations complement the previous work by Woon \& Herbst (2009), who reported the same properties for an ensemble of 200 neutral species. The rationale behind our new calculations is that accurate knowledge of the physicochemical properties of the species in the reaction network databases is a first mandatory step to improving the reliability of the astrochemical models.

All the computed data can be found on the ACO project site. ${ }^{15}$

Finally, we discussed two practical examples to illustrate the potentiality of using our new cations database, coupled with the Woon \& Herbst (2009) one, to identify and exclude endothermic reactions from the astrochemical reaction networks.

This project has received funding within the European Union's Horizon 2020 research and innovation program from the European Research Council (ERC) for the project "The Dawn of Organic Chemistry" (DOC), grant agreement No. 741002, and from the Marie Skłodowska-Curie for the project "AstroChemical Origins" (ACO), grant agreement No. 811312. S.P., N.B., and P.U. acknowledge the Italian Space Agency for cofunding the Life in Space Project (ASI N. 2019-3-U.O). CINES-OCCIGEN HPC is kindly acknowledged for the generous allowance of supercomputing time through the A0060810797 project. Finally, we wish to acknowledge the extremely useful discussions with Professor Gretobape and the LATEX community for the insights on TikZ and PGFPlots packages.

Software: RdKit (Landrum 2016), ASE (Larsen et al. 2017), NetworkX (Hagberg et al. 2008), VMD (Humphrey et al. 1996), JSmol, Gaussian16 (Frisch et al. 2016).

\section{Appendix \\ Extended Internal Coordinates Geometry Optimization Comparison}

An extended internal coordinates geometry optimization comparison is provided in Table 9. The coordinates given in Table 9 follow those expressions in Gaussian16 for specifying generalized internal coordinates. ${ }^{16}$ The complete set of coordinate comparisons is available in a machine-readable format.

Table 9

Extended Internal Coordinates Geometry Optimization Comparison for $1-\mathrm{C}_{3} \mathrm{H}_{2}^{+}$

\begin{tabular}{|c|c|c|c|c|c|c|}
\hline Species & Internal $Z$ Vec. & Internal Coord. & M06-2X/cc-pVTZ & CCSD/aug-cc-pVTZ & $\Delta$ & Unit \\
\hline $1-\mathrm{C}_{3} \mathrm{H}_{2}^{+}$ & $(1,6,6,6,1)$ & $\mathrm{R}(1,2)$ & 1.093 & 1.092 & 0.001 & $\AA$ \\
\hline $1-\mathrm{C}_{3} \mathrm{H}_{2}^{+}$ & $(1,6,6,6,1)$ & $\mathrm{R}(2,3)$ & 1.312 & 1.314 & -0.002 & $\AA$ \\
\hline $1-\mathrm{C}_{3} \mathrm{H}_{2}^{+}$ & $(1,6,6,6,1)$ & $\mathrm{R}(3,4)$ & 1.340 & 1.351 & -0.011 & $\AA$ \\
\hline $1-\mathrm{C}_{3} \mathrm{H}_{2}^{+}$ & $(1,6,6,6,1)$ & $\mathrm{A}(1,2,3)$ & 120.665 & 120.424 & 0.241 & $\AA$ \\
\hline $1-\mathrm{C}_{3} \mathrm{H}_{2}^{+}$ & $(1,6,6,6,1)$ & $\mathrm{A}(1,2,5)$ & 118.903 & 119.111 & -0.208 & $\AA$ \\
\hline $1-\mathrm{C}_{3} \mathrm{H}_{2}^{+}$ & $(1,6,6,6,1)$ & $\mathrm{L}(2,3,4,5,-2)$ & 180.0 & 180.0 & 0 & degrees \\
\hline $1-\mathrm{C}_{3} \mathrm{H}_{2}^{+}$ & $(1,6,6,6,1)$ & $\mathrm{D}(1,2,5,3)$ & 180.0 & 180.0 & 0 & degrees \\
\hline
\end{tabular}

Note. Table 9 is published in its entirety in a machine-readable format. A portion is shown here for guidance regarding its form and content.

(This table is available in its entirety in machine-readable form.)

\footnotetext{
15 aco-itn.oapd.inaf.it/aco-public-datasets/theoretical-chemistry-calculations/ cations-database

${ }^{16}$ Gaussian16 Revision C.01, https://Gaussian.com/gic/.
} 


\section{ORCID iDs}

Lorenzo Tinacci (i) https://orcid.org/0000-0001-9909-9570

Stefano Pantaleone (1) https://orcid.org/0000-0002-2457-1065

Andrea Maranzana (1) https://orcid.org/0000-0002-5524-8068

Nadia Balucani (i) https://orcid.org/0000-0001-5121-5683

Cecilia Ceccarelli (ib https://orcid.org/0000-0001-9664-6292

Piero Ugliengo (i) https://orcid.org/0000-0001-8886-9832

\section{References}

Aue, D. H., \& Bowers, M. T. 1979, Gas Phase Ion Chemistry (Amsterdam: Elsevier)

Bauernschmitt, R., \& Ahlrichs, R. 1996, JChPh, 104, 9047

Bender, H., Carnovale, F., Peel, J. B., \& Wentrup, C. 1988, JAChS, 110, 3458

Berkowitz, J., \& Cho, H. 1989, JChPh, 90, 1

Berkowitz, J., Ellison, G. B., \& Gutman, D. 1994, JPhCh, 98, 2744

Berkowitz, J., Greene, J., Cho, H., \& Ruscić, B. 1987, JChPh, 86, 674

Bieri, G., \& Jonsson, B.-Ö. 1978, CPL, 56, 446

Blush, J. A., \& Chen, P. 1992, JPhCh, 96, 4138

Boo, B. H., \& Armentrout, P. 1987, JAChS, 109, 3549

Bulgin, D. K., Dyke, J. M., \& Morris, A. 1977, J. Chem. Soc., Faraday Trans., 73, 983

Butler, J. J., Holland, D. M., Parr, A. C., \& Stockbauer, R. 1984, IJMSI, 58, 1 Cernicharo, J., Cabezas, C., Bailleux, S., et al. 2021b, A\&A, 646, L7

Cernicharo, J., Cabezas, C., Endo, Y., et al. 2021a, A\&A, 646, L3

Chabot, M., Béroff, K., Gratier, P., Jallat, A., \& Wakelam, V. 2013, ApJ, 771,90

Chase, M. W. 1996, JPCRD, 25, 551

Cheung, A., Rank, D. M., Townes, C., Thornton, D. D., \& Welch, W. 1968, PhRvL, 21, 1701

Cheung, A., Rank, D. M., Townes, C., Thornton, D. D., \& Welch, W. 1969, Natur, 221, 626

Clauberg, H., Minsek, D. W., \& Chen, P. 1992, JAChS, 114, 99

Clemmer, D., \& Armentrout, P. 1992, JChPh, 97, 2451

Cockett, M. C., Dyke, J. M., Morris, A., \& Niavaran, M. H. Z. 1989, J. Chem. Soc., Faraday Trans., 85, 75

Dibeler, V. H., \& Liston, S. K. 1968, JChPh, 48, 4765

Douglas, J. E., Rabinovitch, B. t., \& Looney, F. 1955, JChPh, 23, 315

Drowart, J., Smets, J., Reynaert, J., \& Coppens, P. 1978, Adv. Mass Spectrom. A, 7, 647

Dyke, J., Dunlavey, S., Jonathan, N., \& Morris, A. 1980, MolPh, 39, 1121

Dyke, J. M. 1987, J. Chem. Soc., Faraday Trans., 83, 69

Dyke, J. M., Morris, A., \& Ridha, A. 1982, J. Chem. Soc., Faraday Trans., 78, 2077

Dyke, J. M., Morris, A., \& Trickle, I. R. 1977, J. Chem. Soc., Faraday Trans., 73, 147

Erman, P., Karawajczyk, A., Rachlew-Källne, E., et al. 1993, CPL, 215, 173

Frisch, M. J., Trucks, G. W., Schlegel, H. B., et al. 2016, Gaussian Revision B.01 (Wallingford, CT: Gaussian, Inc.)

Frost, D., Lee, S., \& McDowell, C. 1973, CPL, 23, 472

Gochel-Dupuis, M., Delwiche, J., Hubin-Franskin, M.-J., \& Collin, J. 1992, CPL, 193, 41

Hagberg, A. A., Schult, D. A., \& Swart, P. J. 2008, Proc. 7th Python in Science Conf., ed. G. Varoquaux, T. Vaught, \& J. Millman, 11, http://conference. scipy.org/proceedings/scipy2008/paper_2/

Hepburn, J., Trevor, D., Pollard, J., Shirley, D., \& Lee, Y. 1982, JChPh, 76,4287

Herbst, E., \& Klemperer, W. 1973, ApJ, 185, 505

Herzberg, G. 1966, Electronic Spectra and Electronic Structure of Polyatomic Molecules, Vol. 3 (New York: Van Nostrand)

Holmes, J., Lossing, F., \& Mayer, P. 1993, CPL, 212, 134

Huber, K.-P. 2013, Molecular Spectra and Molecular Structure: IV. Constants of Diatomic Molecules (Berlin: Springer Science \& Business Media)

Humphrey, W., Dalke, A., \& Schulten, K. 1996, J. Mol. Graph., 14, 33

Kendall, T., Jr 1992, JChPh, 96, 6796
Klemperer, W. 1970, Natur, 227, 1230

Knowles, P. J., Hampel, C., \& Werner, H.-J. 1993, JChPh, 99, 5219

Landrum, G. 2016, RDKit: Open-Source Cheminformatics Software, https:// www.rdkit.org/

Larsen, A. H., Mortensen, J. J., Blomqvist, J., et al. 2017, JPCM, 29, 273002

Lattelais, M., Pauzat, F., Ellinger, Y., \& Ceccarelli, C. 2009, ApJL, 696, L133

Lattelais, M., Pauzat, F., Ellinger, Y., \& Ceccarelli, C. 2010, A\&A, 519, A30

Lias, S. G., Bartmess, J. E., Liebman, J., Holmes, J., \& Levin, R. D. 1988, JPCRD, 17, Suppl. 1, https://srd.nist.gov/JPCRD/jpcrdS1Vol17.pdf

McGuire, B. A., Asvany, O., Brünken, S., \& Schlemmer, S. 2020, NatRP, 2, 402

Milan, J., Buma, W., \& De Lange, C. 1996, JChPh, 104, 521

Nagano, Y., Murthy, S., \& Beauchamp, J. 1993, JAChS, 115, 10805

Nakasgawa, H., Asano, M., \& Kubo, K. 1981, JNuM, 102, 292

Norwood, K., \& Ng, C. 1989a, CPL, 156, 145

Norwood, K., \& Ng, C. 1989b, JChPh, 91, 2898

Ohno, K., Okamura, K., Yamakado, H., et al. 1995, JPhCh, 99, 14247

Plessis, P., \& Marmet, P. 1986, IJMSI, 70, 23

Plessis, P., \& Marmet, P. 1987, CaJCh, 65, 2004

Podio, L., Codella, C., Lefloch, B., et al. 2017, MNRAS, 470, L16

Qi, F., Sheng, L., Zhang, Y., Yu, S., \& Li, W.-K. 1995, CPL, 234, 450

Ramanathan, R., Zimmerman, J. A., \& Eyler, J. R. 1993, JChPh, 98, 7838

Rappé, A. K., Casewit, C. J., Colwell, K., Goddard, W. A., III, \& Skiff, W. M. 1992, JAChS, 114, 10024

Reineke, W., \& Strein, K. 1976, Berichte der Bunsengesellschaft für physikalische Chemie, 80, 343

Reiser, G., Habenicht, W., Müller-Dethlefs, K., \& Schlag, E. W. 1988, CPL, 152,119

Rohlfing, E. A., Cox, D. M., \& Kaldor, A. 1984, JChPh, 81, 3322

Rosi, M., Mancini, L., Skouteris, D., et al. 2018, CPL, 695, 87

Rosi, M., Skouteris, D., Balucani, N., et al. 2019, International Conference on Computational Science and Its Applications (Berlin: Springer), 306

Ruscic, B., \& Berkowitz, J. 1993, JChPh, 98, 2568

Ruscic, B., \& Berkowitz, J. 1994, JChPh, 100, 4498

Ruscic, B., Berkowitz, J., Curtiss, L., \& Pople, J. 1989, JChPh, 91, 114

Shavitt, I. 1985, Tetrahedron, 41, 1531

Shin, S. K., Corderman, R. R., \& Beauchamp, J. 1990, IJMSI, 101, 257

Shiner, D., Gilligan, J., Cook, B., \& Lichten, W. 1993, PhRvA, 47, 4042

Smoes, S., Myers, C., \& Drowart, J. 1971, CPL, 8, 10

Snow, K. B., \& Thomas, T. F. 1990, IJMSI, 96, 49

Snyder, L., Le, S., \& Jm, H. 1976, Reprints: Series A, Vol. 212 (Green Bank, WV: National Radio Astronomy Observatory), 383

Snyder, L. E., Buhl, D., Zuckerman, B., \& Palmer, P. 1969, PhRvL, 22, 679

Tang, X., Lin, X., Garcia, G. A., et al. 2020, JChPh, 153, 124306

Tao, W., Klemm, R., Nesbitt, F., \& Stief, L. 1992, JPhCh, 96, 104

Tercero, B., Vincent, L., Cernicharo, J., Viti, S., \& Marcelino, N. 2011, A\&A, 528, A26

Tiedemann, P., Anderson, S., Ceyer, S., et al. 1979, JChPh, 71, 605

Tonkyn, R. G., Winniczek, J. W., \& White, M. G. 1989, CPL, 164, 137

Traeger, J. C. 1985, IJMSI, 66, 271

Traeger, J. C., McLoughlin, R. G., \& Nicholson, A. 1982, JAChS, 104, 5318

Trickl, T., Cromwell, E., Lee, Y., \& Kung, A. 1989, JChPh, 91, 6006

Trinajstic, N. 2018, Chemical Graph Theory (London: Routledge)

Verhaegen, G., Stafford, F., \& Drowart, J. 1964, JChPh, 40, 1622

Vogt, J., Williamson, A. D., \& Beauchamp, J. 1978, JAChS, 100, 3478

Wakelam, V., Herbst, E., Loison, J.-C., et al. 2012, ApJS, 199, 21

Walters, E., \& Blais, N. C. 1984, JChPh, 80, 3501

Wang, L.-S., Reutt, J., Lee, Y., \& Shirley, D. 1988, JESRP, 47, 167

Wang, R.-G., Dillon, M., \& Spence, D. 1984, JChPh, 80, 63

Watson, W. D. 1973, ApJL, 183, L17

Watts, J. D., Gauss, J., \& Bartlett, R. J. 1993, JChPh, 98, 8718

Wiedmann, R., Tonkyn, R., White, M., Wang, K., \& McKoy, V. 1992, JChPh, 97,768

Wlodek, S., \& Bohme, D. K. 1989, J. Chem. Soc., Faraday Trans., 85, 1643

Woon, D. E., \& Dunning, T. H., Jr 1993, JChPh, 98, 1358

Woon, D. E., \& Herbst, E. 2009, ApJS, 185, 273

Zanchet, A., Roncero, O., Agúndez, M., \& Cernicharo, J. 2018, ApJ, 862, 38

Zhao, Y., \& Truhlar, D. G. 2008, Theor. Chem. Acc., 120, 215 